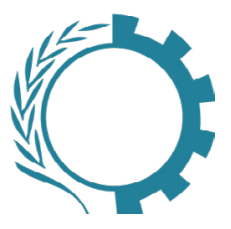

\title{
Social Network Analysis Approach to Analysis of Emergency Management Team Performance
}

Marzieh Abbassinia, PhD in Occupational Health and Safety, Center of Excellence for Occupational Health, Occupational Health and Safety Research Center, School of Public Health, Hamadan University of Medical Sciences, Hamadan, Iran.

Omid Kalatpour, Assistant professor, Center of Excellence for Occupational Health, Occupational Health and Safety Research Center, School of Public Health, Hamadan University of Medical Sciences, Hamadan, Iran.

Majid Motamedzade, Professor, Department of Ergonomics, School of Public Health in Hamadan University of Medical Sciences.

Alireza Soltanian, Professor, department of Biostatistics, Modeling of Noncommunicable Diseases Research Center, Hamadan University of Medical Sciences, Hamadan, Iran.

D Iraj Mohammadfam, ( ${ }^{*}$ Corresponding author), Professor, center of Excellence for Occupational Health, Occupational Health and Safety Research Center, School of Public Health, Hamadan University of Medical Sciences, Hamadan, Iran. mohammadfam@umsha.ac.ir

Mohammad Ganjipour, Head of improvement procedures and process analysis and secretary of system suggestions, Shazand Petrochemical Company, Arak, Iran.

Leila Khodabandehloo, Msc, Department of Ergonomics, University of Social Welfare and Rehabilitation Science, Tehran, Iran.

\section{Abstract}

Since emergencies are often unpredictable, organizations need to be prepared to overcome the threats. In addition to emergency teams, coordination and preparedness also is very important in responding to emergencies. This study aimed to use social network analysis to improve the performance of an emergency management system in the petrochemical industry. The studied emergency was, hydrogen leak from the cylinder joints in the olefin unit. Social Network Analysis (SNA) was used to evaluate the emergency team's response. To collect the required information, emergency scenarios, questionnaires and interviews with experts and specialists was done. Gephi software version 0.9.1 was used to analyze the social network data of this study. The findings of this study indicates a high disruption between members and low network density. The network density index was 0.108 , which shows the low coherence of the network and a significant gap between the members. Meaning that only $10.8 \%$ of all the possible connections within the emergency management members were established. Low density leads to inconsistency, lack of cooperation and poor performance in emergency management. On average, the study showed that, the manager of operations had the most influence on the network. The social network analysis approach, helps managers and decision makers to identify the strengths and weaknesses of an emergency response team, and focus on the interactions and relationships between the teams, as well as their coordination. Also, to achieve optimal coordination in the emergency response, it is necessary to consider specific plans and instructions, and the identification of the tasks.

\section{Conflicts of interest: None}

Funding: Hamadan University of Medical Sciences.

\section{Keywords \\ Crisis Management \\ Social Network Analysis}

Emergencies

Received: 2020/09/06

Accepted : 2021/05/29 
Introduction

Crises and emergencies are generally unexpected, unplanned and serious situations, in which the organization requires an immediate action and intervention to prevent the worsening of the situation (1). So it is necessary to have an efficient emergency management system designed to appropriately respond to emergencies $(2,3)$. Despite all the efforts to reduce the negative effects of emergencies and crises, these situations are having an effect on an individual, group, community and even the whole society (4, 5 ). In the absence of a fast and immediate response to an emergency, there is a potential expansion and intensification of this effects. The petrochemical industry, is one of the high risk industries in which, accidents, especially fire and explosions in the petrochemical companies, are still causing great losses (1). Since crises and emergencies are often unpredictable, in order to be able to overcome the threats, organizations and individuals need to be prepared to face any emergency situation (6). In this regard, a standardized emergency management system, will reduce the costs, mitigates the environmental and human impacts, reduces the recovery time, and preserves the corporate's integrity. Well established emergency management plans, help to organize and manage the resources to reduce or prevent all the negative aspects of an emergency $(5,7)$. These emergency plans are usually predefined to ensure the implementation of rapid, systematic and effective emergency measures (7). This system includes individuals, groups, equipment, coordinations and the relationship between individuals and groups. Emergency management is performed by more than one person and there are different teams and individuals managing these situations, each with specific duties and responsibilities during the emergency (8). Emergency personnel and teams alone, are not enough for an effective response to emergencies, and the preparedness and coordination of these individuals and teams as a network, and the sustainability of this network in emergencies is very important (9). Coordination has always been a challenge in emergency management. Social network analysis is one of the most common tools for examining preparedness and coordination amongst individuals and teams within a network. Studies have shown that SNA tools, that are used to examine relationships during emergencies and crises, helps to better understand the performance, and the strengths and weaknesses of the teams (10). This method can provide useful information, for decision makers about the performance of individuals, as well as how team members communicate to each other in the network (11). SNA indicators can be used to identify groups and individuals, with the least and the most importance in the network, identify the strengths and weaknesses of the emergency response teams, and making the necessary changes to improve the response. This study is aimed to use social network analysis, to improve the performance of an emergency management system in the petrochemical industry.

\section{Methodology}

The studied emergency was, the hydrogen leakage from the cylinder joints in the olefin unit. Social Network Analysis (SAN) approach, was used to evaluate intra- and intergroup responses in emergency management teams, identifying the key roles, identifying the leadership roles in different groups, identifying groups and individuals with a greater degree of centrality and the required changes to achieve the highest performance rate. Social Network Analysis, typically consist of an actor who receives or sends some sort of information or resources, and the network allows for the exchange of this information or resources. Social Network Analysis is a developed form of graph theory and provides a mathematical description of the relationship between two sets: the set of nodes and the set of edges, which form a network when combined. The nodes are persons, groups, or organizations, and the ties are the relationship between these entities that consist of a wide range of collaborations and interpersonal communications (12). In Social Network Analysis, these relationships are represented via graphs. Studying emergency scenarios, questionnaires, and interviews with experts and specialists were used to collect the required information for social networks analysis, about the individuals and groups involved in the emergency management. Gephi software version 0.9.1 was used to analyze the social network data of this study. The emergency management team of the studied petrochemical company, consisted of 7 groups and 79 individuals. These teams were: the management, fire department, operation, security, HSE, medical, and one person as a commander. The studied emergency was hydrogen leakage from the cylinder joints in the olefin unit. Hydrogen is the lightest element in the periodic table with a high probability of catching fire or causing an explosion (13). 22 tanks of hydrogen was in the hydrogen storage section, which were designed to supply the hydrogen tanks. Hydrogen storage is one of the most sensitive and dangerous sections in the complex, due to storing a significant volume of hydrogen under high pressures. Immediately after the emergency occurred, emergency management team's reacted, to reduce any injuries or losses. Teams and individuals that are participating in the emergency management team, will take actions based on the tasks that were specified in the scenario. Emergency communication cycle at the beginning of the study are presented in Figure 1. 


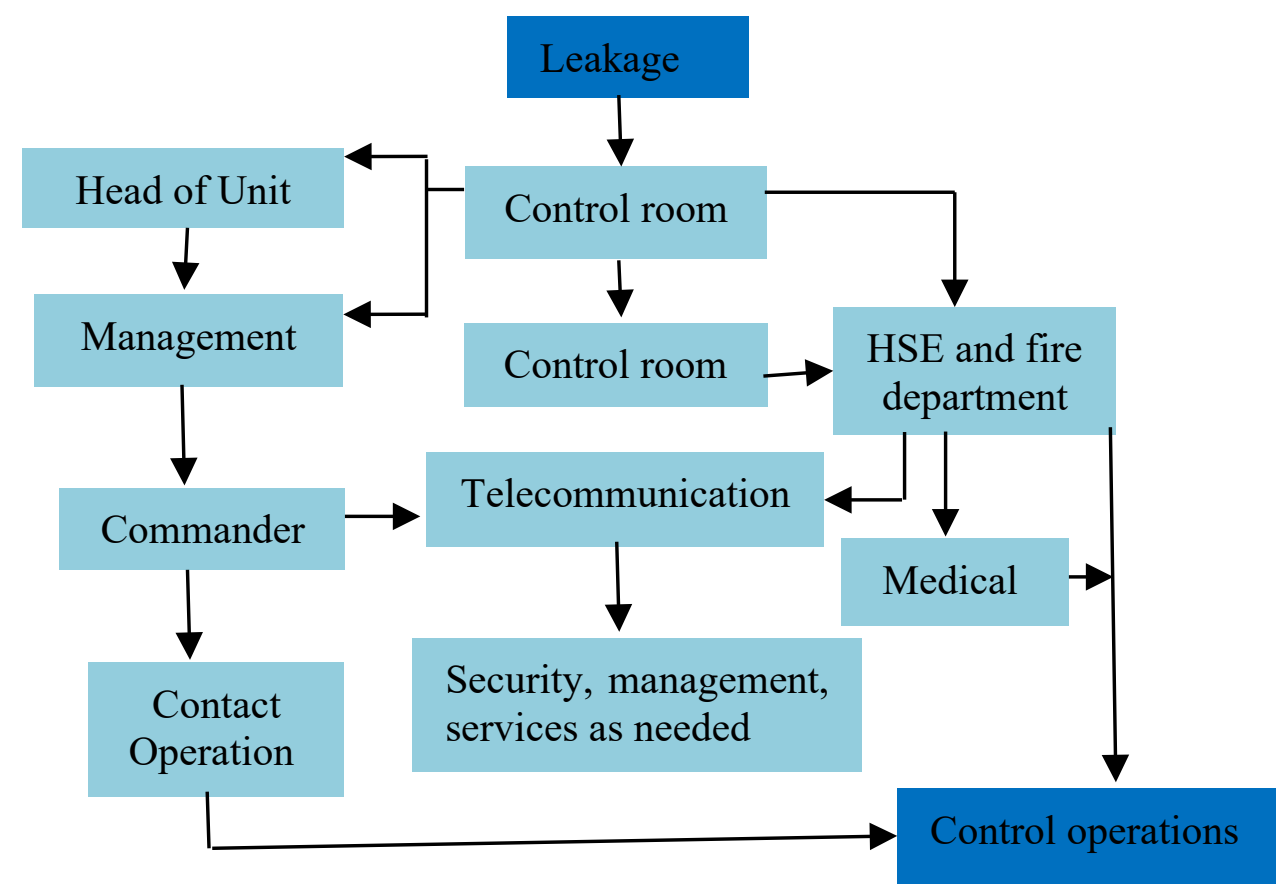

Figure 1. Communication Graph at the beginning of the studied emergency situation

Result

The findings of this study indicates a high disruption between team members and a low network density. The network density index was 0.108 , which represents a significant gap between the members and the low coherence of the network. Meaning that only $10.8 \%$ of all the possible connections between the members of the emergency management group were established. Based on the studies, individuals who were embedded in relatively dense networks, had a better communication and interaction, compared to the individuals in a less-dense network. So, the low density leads to inconsistency between individuals and teams, and ultimately leads to the lack of cooperation and the poor performance in the emergency management and crisis teams. Each member of the emergency management team, was considered as a node in the network, and their interactions and their relationships were examined as edges. The groups involved in emergencies and their tasks are shown in Table 1.

Examining the degree of centrality in the network, shows that the operation's commander has the highest degree of centrality in the network. Senior management had the second highest degree of centrality in the network, followed by management of the fire department. The network characteristics are showed in Table 2.

According to the table 2, the highest density was related to the commander of the operation and the management, which indicates the maximum communication and interactions in these two groups. The operation group also had the most density. The highest degree of centrality, was obtained by the commander of the operations and the management, which indicates that these two groups, have the greatest impact on the entire network. The lowest average degree of centrality, was obtained by the security group, which indicates the lowest impact level, this group had on the entire network. The Commander of the operations and the management also had the highest, average betweenness centrality, as well as the highest average of closeness centrality. The lowest amounts of these two indicators, was obtained by the security group.

\section{Discussion}

In addition to focusing on individuals, social network analytics approach, helps the managers and decision makers to identify the strengths and weaknesses of an emergency response team, and focus on the interactions and relationships between teams and their coordination level (13). Based on the weakness in the density of the network, we conclude that, if the involvement of the individuals and the teams improved within the network, their ability to coordinate will also increase. Coordination and interactions are especially important in teams that have a direct operational role in the emergency management (8). Using coordination assessment based on network connectedness, an organization can be reviewed in order to identify the current state 
Abbassinia M, et al.

Table 1. Groups involved in emergencies and their tasks

\begin{tabular}{|c|c|}
\hline Groups & Tasks \\
\hline Management & $\begin{array}{l}\text { Announcing the state of an emergency, informing and monitoring the performance, announcing the } \\
\text { required instructions to all departments, assessing the need of evacuate units, assessing the need to attract } \\
\text { external assistance, etc. }\end{array}$ \\
\hline Commander & $\begin{array}{l}\text { Necessary coordination to announce and departure groups to the accident site for cooperation and } \\
\text { coordination. }\end{array}$ \\
\hline Fire Stations & $\begin{array}{l}\text { Identify the location and departure to extinguish, Ensure emergency exit routes are open, provide PPE to } \\
\text { other teams, and consider the supportive measures for the other teams. }\end{array}$ \\
\hline Operations & $\begin{array}{l}\text { Isolation of the area, monitoring the condition of the injured, providing needed equipment, assistance to } \\
\text { injured before the arrival of the ambulance, and resolving any possible problems at the scene of the accident. }\end{array}$ \\
\hline HSE & $\begin{array}{l}\text { Safety command, recognize and report situations of risk, installation of safety signs, control of firefighting } \\
\text { operations, organizing evacuation and enclosing danger, monitoring the performance of } \mathrm{H}, \mathrm{S}, \mathrm{E} \text {, organizing } \\
\text { the dispatch of rescue teams, coordinating with the medical, Monitoring the existence of the appropriate } \\
\text { and sufficient firefighting equipment, decontamination, constant monitoring of the environment in terms } \\
\text { of dangerous situations. }\end{array}$ \\
\hline Security & $\begin{array}{l}\text { Security, control the locations leading up to the accident site, traffic control, coordination with the police, } \\
\text { assistance in evacuation, establishing law and order in the area, leading rescue groups, installing safety tapes. }\end{array}$ \\
\hline Medical & Patient care at the scene of the accident as well as hospitalizing the patients in the hospital. \\
\hline
\end{tabular}

Table 2. Features of the examined network

\begin{tabular}{|c|c|c|c|c|c|c|}
\hline Group & $\begin{array}{l}\text { Number } \\
\text { of nodes }\end{array}$ & $\begin{array}{l}\text { Number } \\
\text { of edges }\end{array}$ & $\begin{array}{l}\text { Average } \\
\text { density }\end{array}$ & $\begin{array}{c}\text { Average degree } \\
\text { of centrality }\end{array}$ & $\begin{array}{c}\text { Average betweenness } \\
\text { centrality }\end{array}$ & $\begin{array}{c}\text { Average closeness } \\
\text { centrality }\end{array}$ \\
\hline Management & 5 & 11 & 1 & 16.4 & 125.7 & 0.47 \\
\hline Commander & 1 & & 1 & 35 & 761 & 0.6 \\
\hline Fire Stations & 17 & 67 & 0.493 & 9.88 & 30.01 & 0.4 \\
\hline Operations & 21 & 51 & 0.243 & 6.38 & 66.51 & 0.381 \\
\hline HSE & 12 & 41 & 0.621 & 8.75 & 39.2 & 0.397 \\
\hline Security & 10 & 13 & 0.289 & 4.7 & 26.63 & 0.273 \\
\hline Medical & 13 & 37 & 0.47 & 7.1 & 32.07 & 0.385 \\
\hline
\end{tabular}

of connectedness and can therefore be judged for its potential to coordinate in an emergency and crisis. Also, to achieve the optimal coordination level in an emergency response, it is necessary to consider specific plans and instructions, precise identification of the tasks and details of the organizational communication process.

\section{Acknowledgment}

This study is supported by the Hamadan University of Medical Sciences, Iran (grant No. 9611037026).

\section{Conflict of Interest}

The authors declares, that there are no conflicts of interest regarding the publication of this manuscript.

\section{How to cite this article:}

Marzieh Abbassinia, Omid Kalatpour, Majid Motamedzade, Alireza Soltanian, Iraj Mohammadfam, Mohammad Ganjipour, Leila Khodabandehloo. Social Network Analysis Approach to Analysis of Emergency Management Team Performance. Iran Occupational Health. 2021 (01 Apr);18:7. 


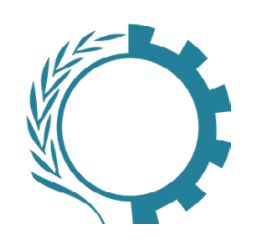

مقاله يخوهشى

http://ioh.iums.ac.ir

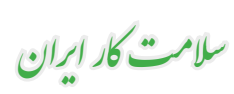

$V / I f \cdots-110$.

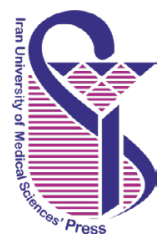

\section{ارزيابى عملكر د تيم هاى واكثش در شر ايط اضطر ارى با استفاده از رويكر ث تحليل شبكه اجتماعى}

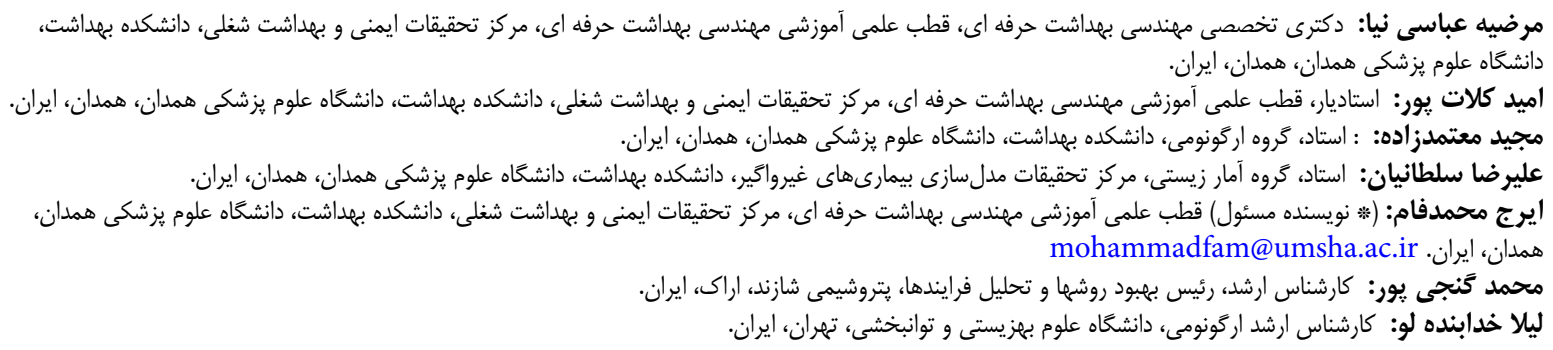

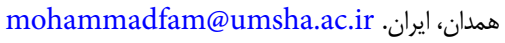

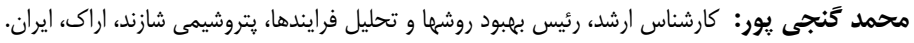

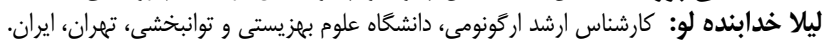

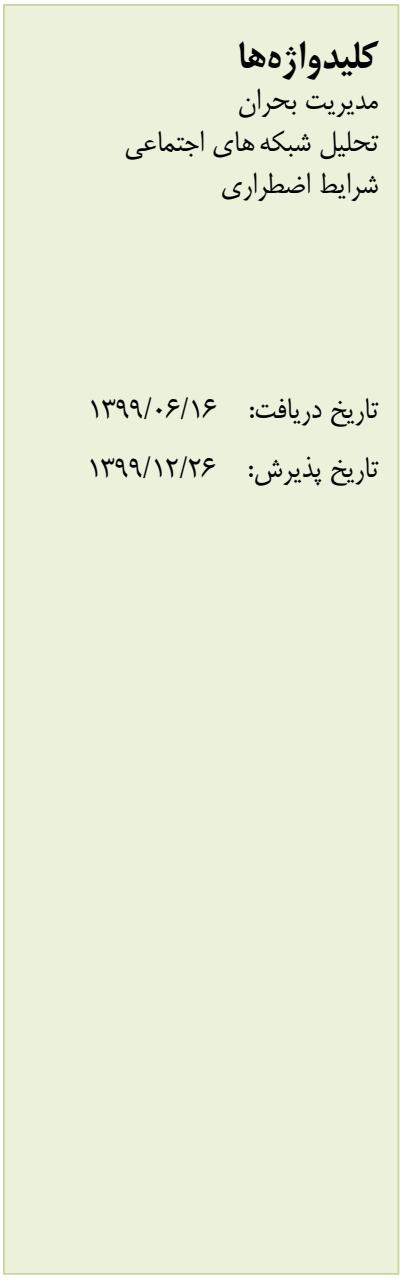

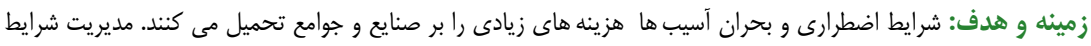

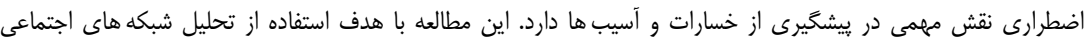

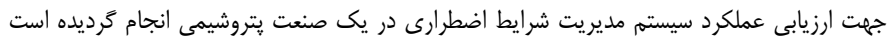

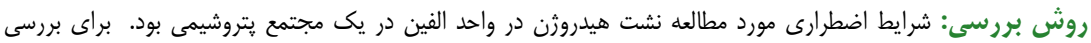

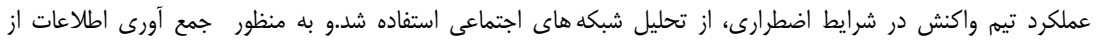

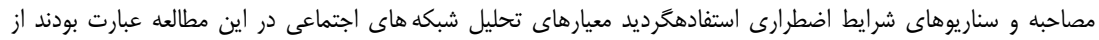

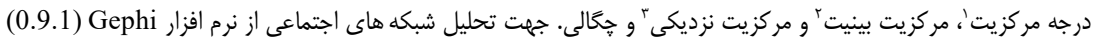 استفاده شد.

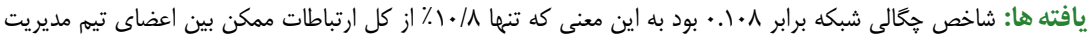

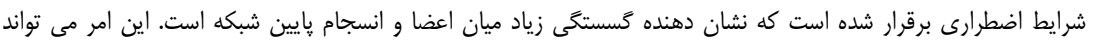

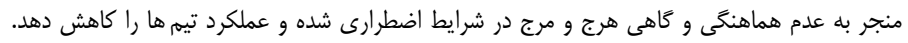
نتيجه تَيرى: بكاركيرى روش تحليل شبكه هاى اجتماعى به مديران و تصميم كيرندكان كمكى مى كندان نقاط ضعف و و

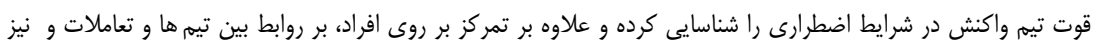 هماهنكى آنها توجه نمايند. تعارض منافع: كزارش نشده /ست.

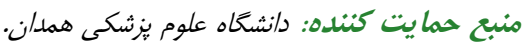

1 Degree of centrality

2 Betweenness centrality

3 Closeness Centrality

شيوه استناد به اين مقاله:

Marzieh Abbassinia, Omid Kalatpour, Majid Motamedzade, Alireza Soltanian, Iraj Mohammadfam, Mohammad Ganjipour, Leila Khodabandehloo. Social Network Analysis Approach to Analysis of Emergency Management Team Performance. Iran Occupational Health. 2021 (01 Apr);18:7. 
روبرو و احتمال شكست در اجراى برنامه هاى مديريت

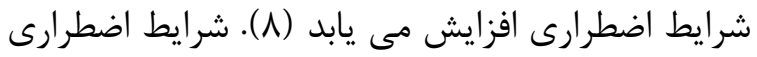

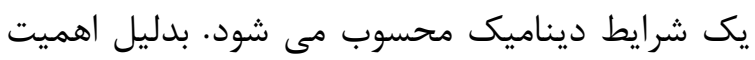

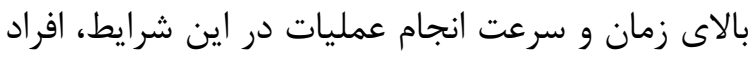

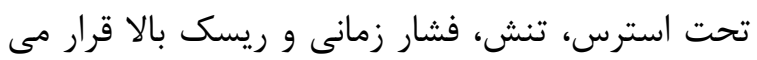

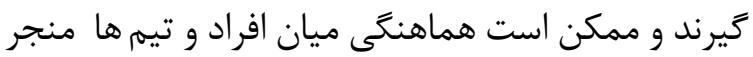

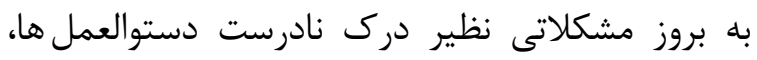

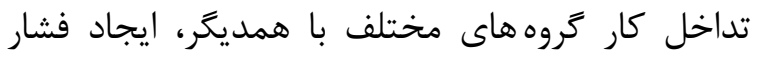

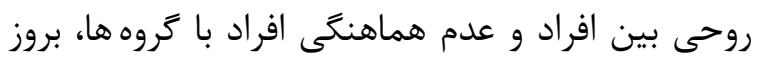

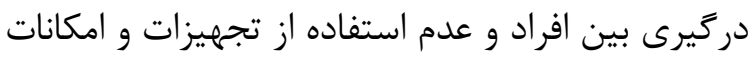

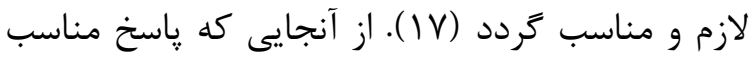

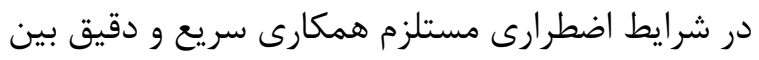

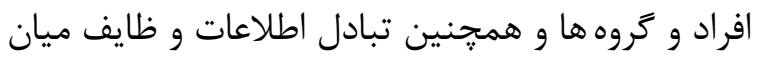

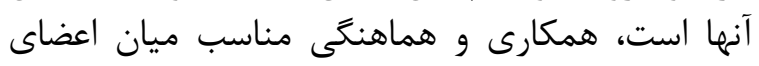

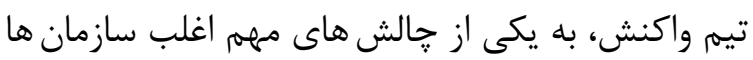

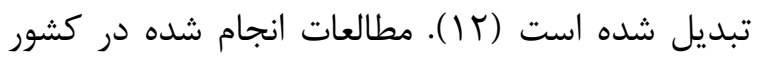

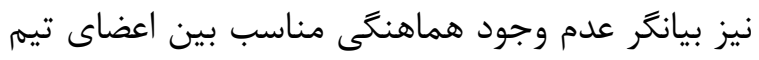

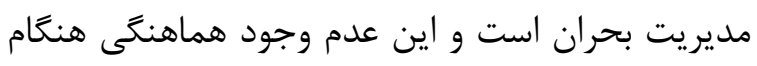

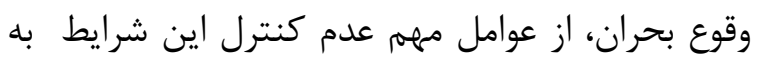

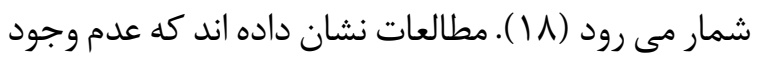

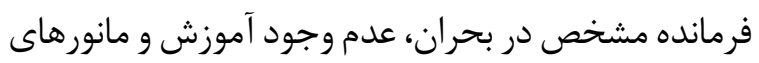

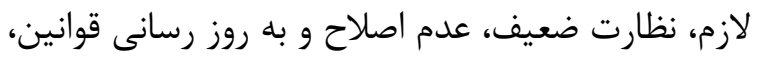

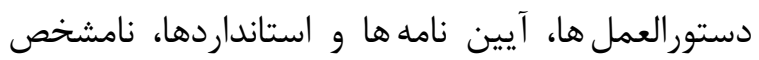

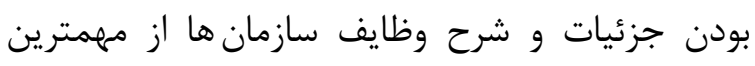

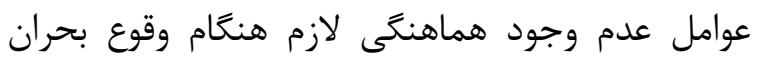
است (19). هدف از ايجاد هماهنكى ميان تيمه ها، ايجاد

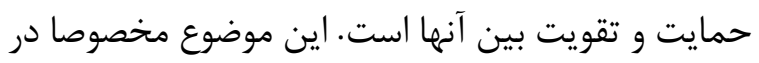

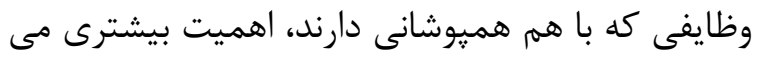

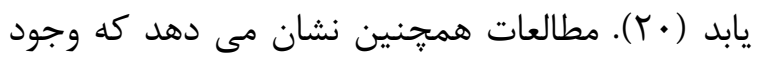

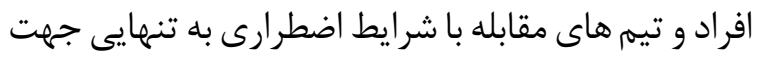

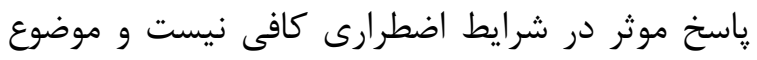

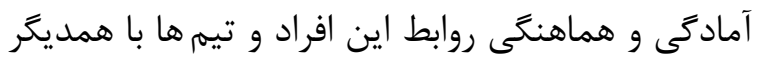

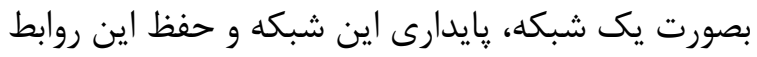

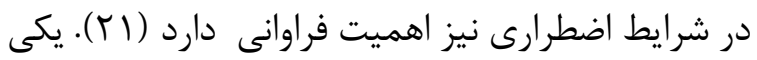

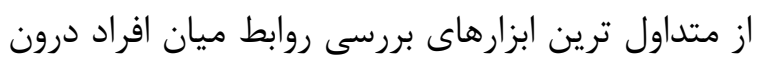

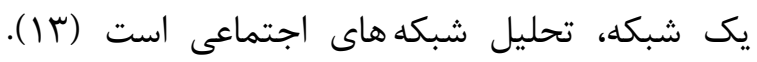

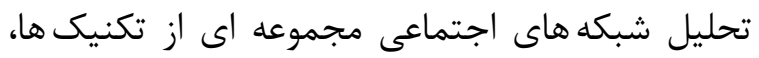

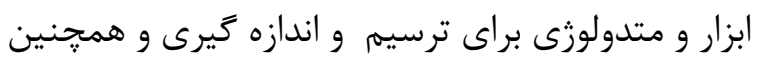

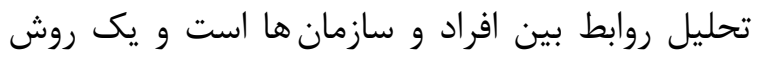

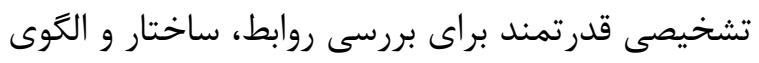

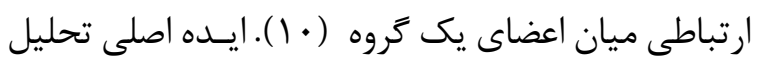

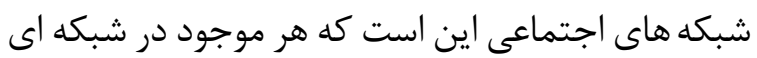

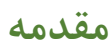

در صنعت يتروشيمى اغلب محصولات و مواد اوليه و

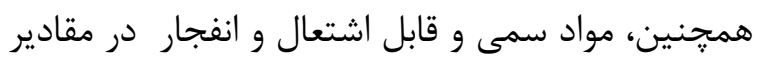

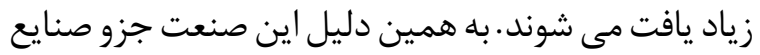

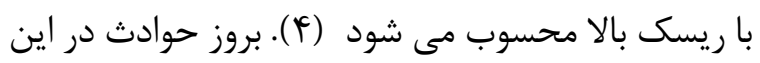

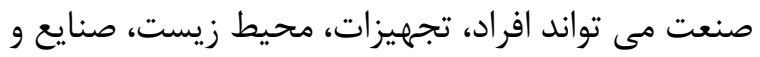

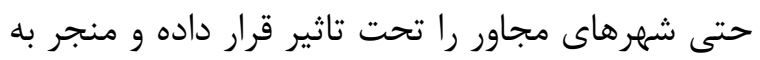

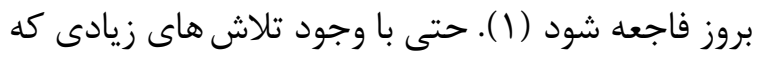

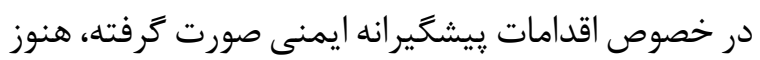

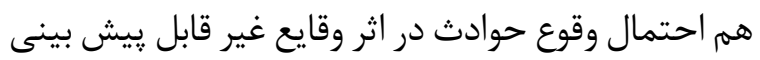

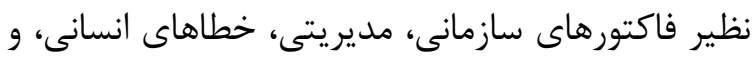

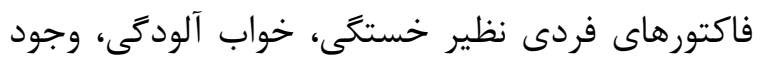

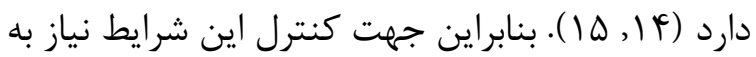

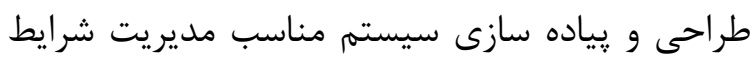

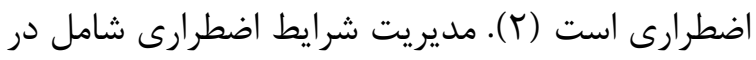

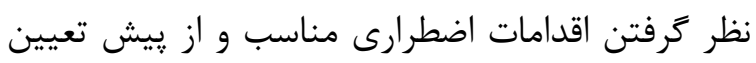

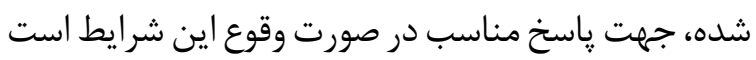

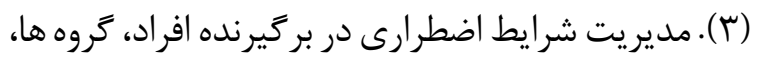

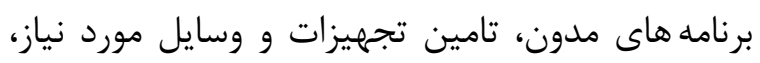

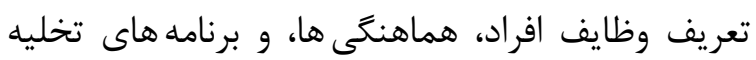

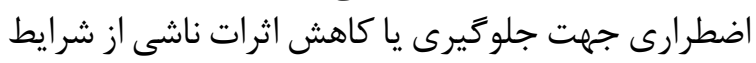

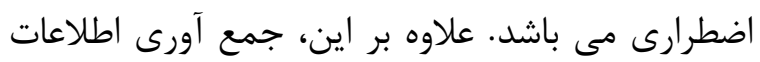

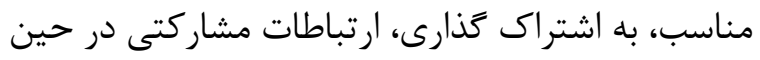

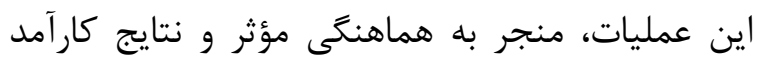

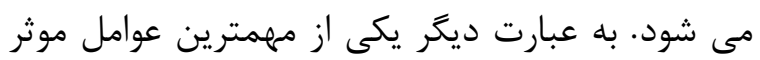

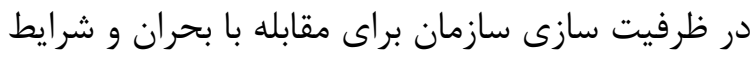

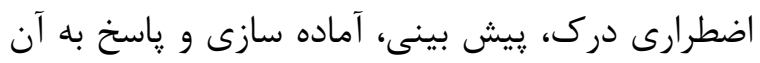

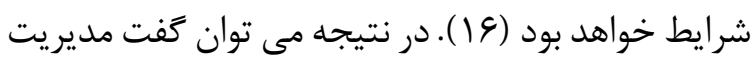

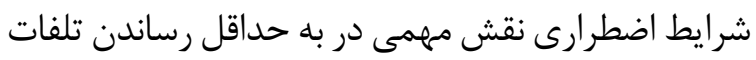

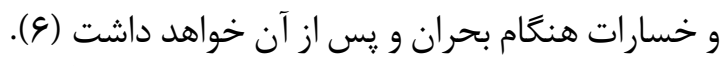

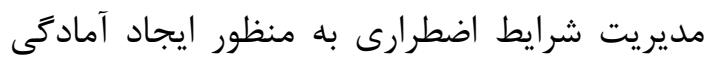

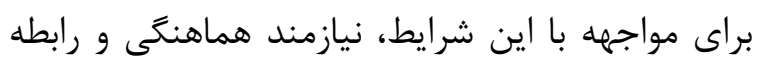

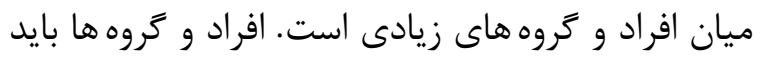

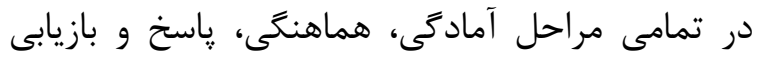

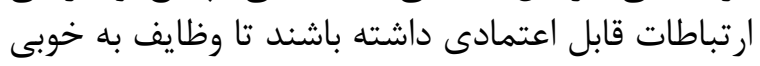

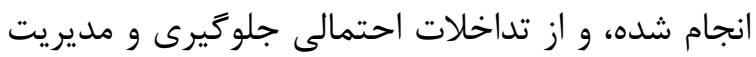

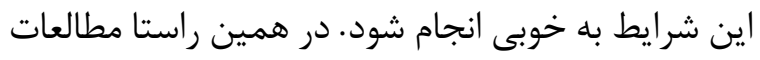

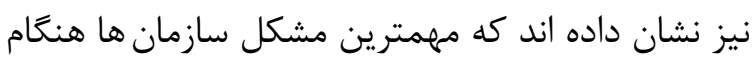

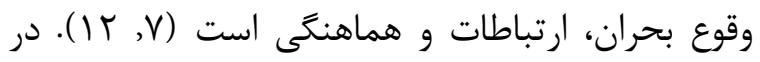

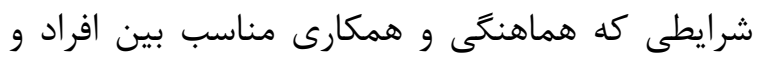

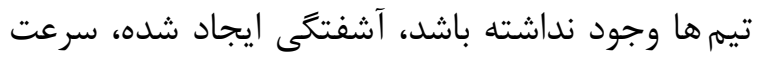
عمل كاهش يافته، مديريت شرايط بن با مشكلات جدى آثلى شرع 


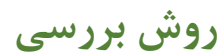

در اين مطالعه از تحليل شبكه هاى اجتماعى جهت

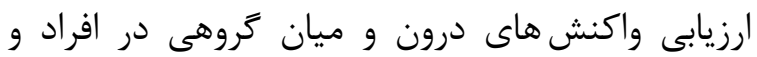

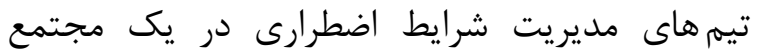

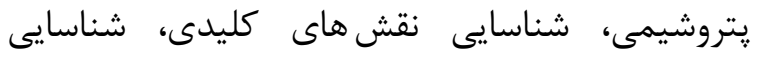

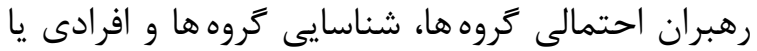

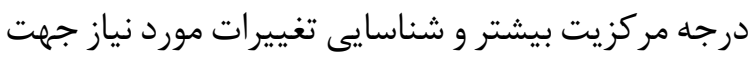

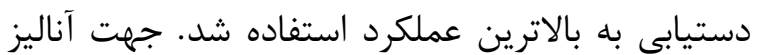

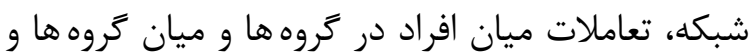

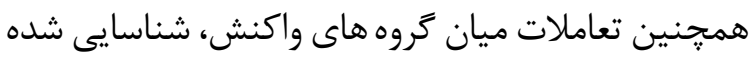

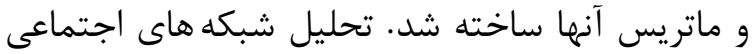

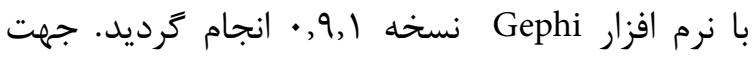

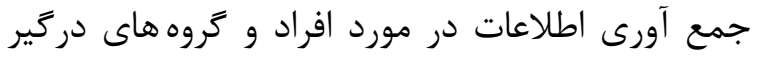

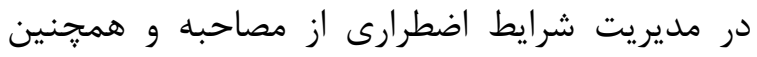

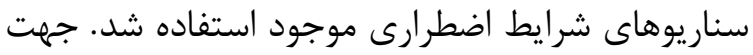

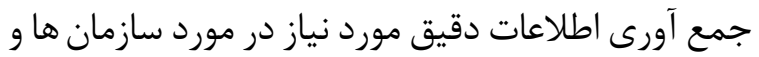

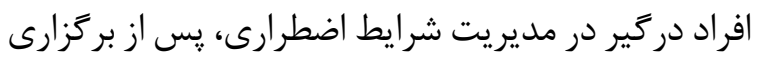

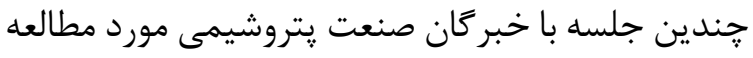

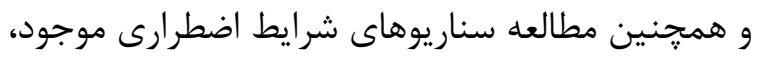

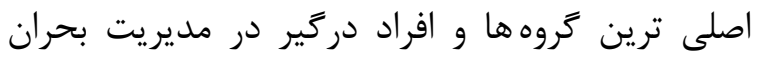

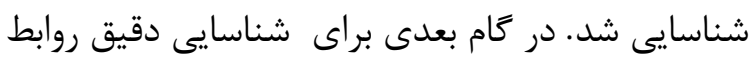

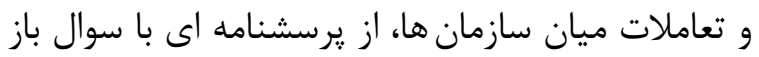

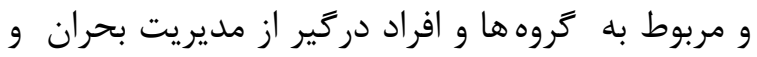

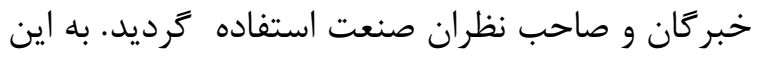

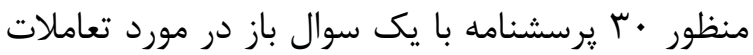

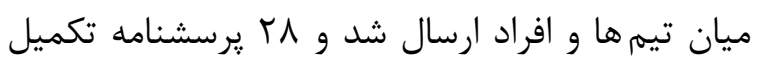

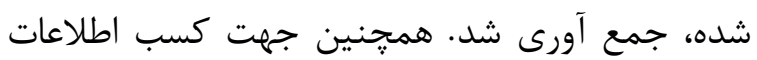

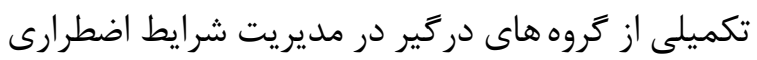

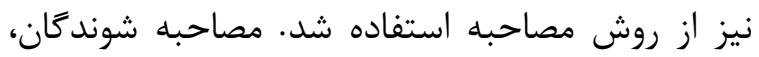

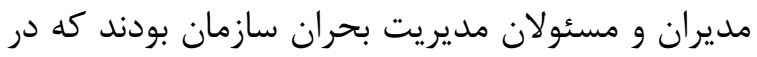

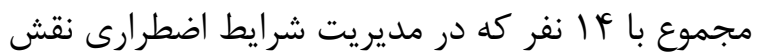

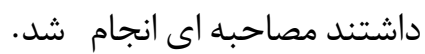

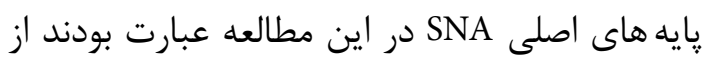

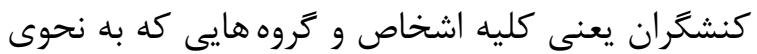

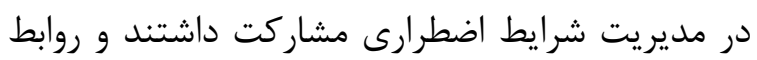

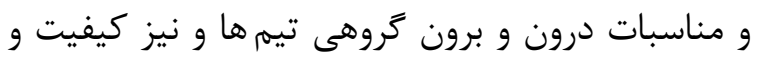

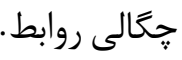

معيارهاى مورد استفاده در اين مطالعه عبارت بودند ازئ

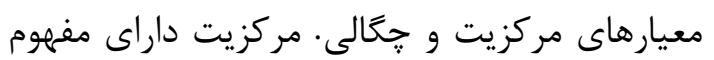

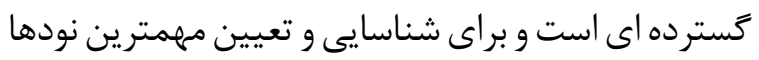

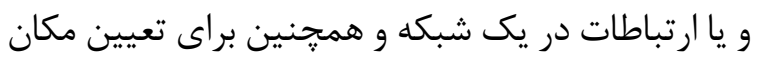

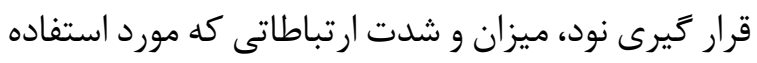

با موجودات ديخر ارتباط دارد. اين ارتباط مى تواند نشان

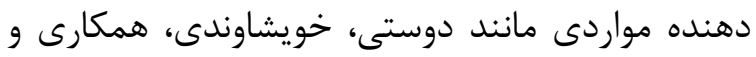

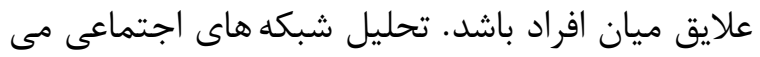

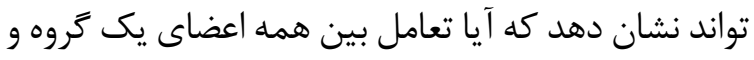

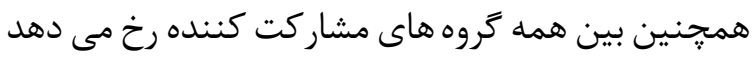

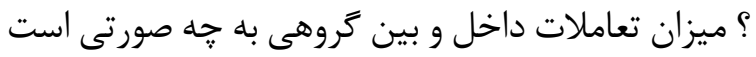

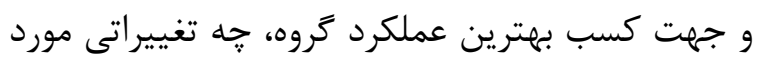

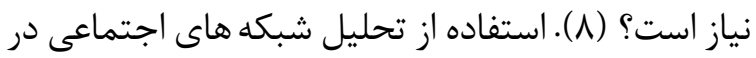

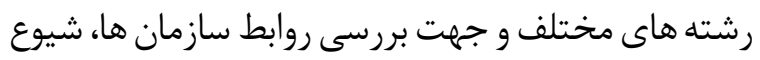

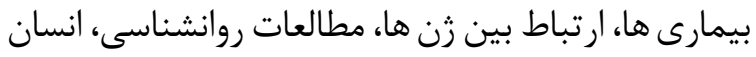

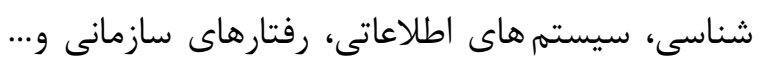

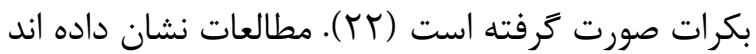

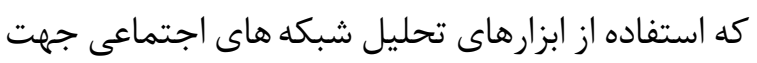

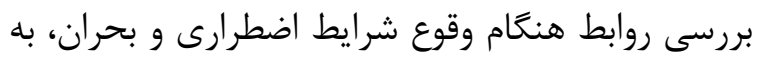

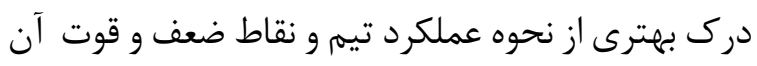

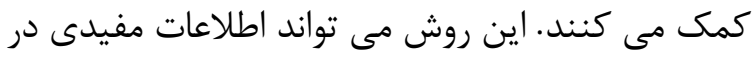

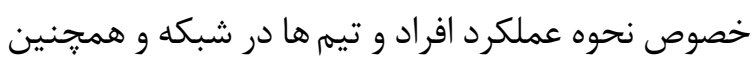

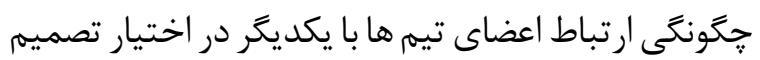

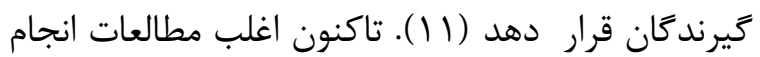

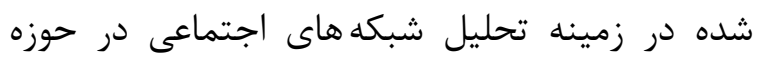

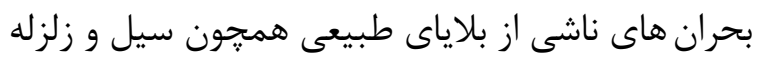

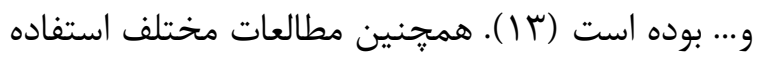

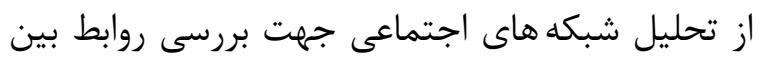

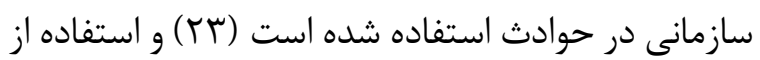

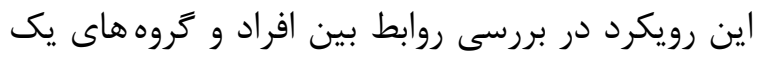

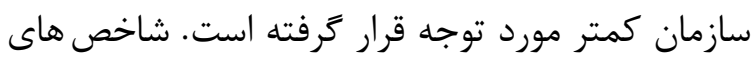

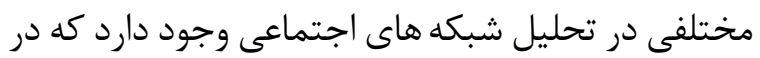

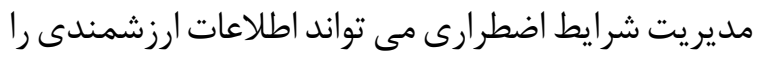

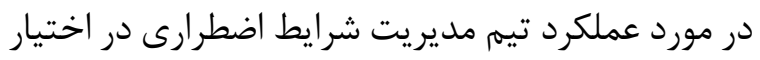

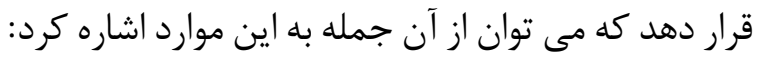

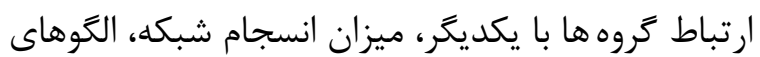

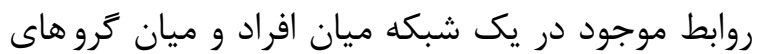

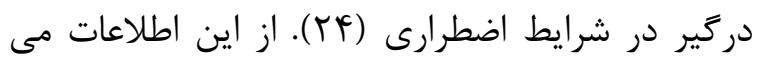

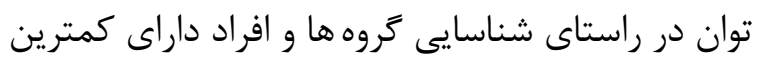

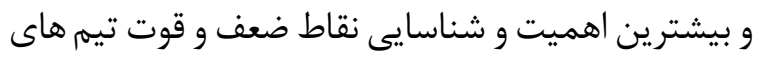

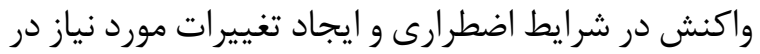

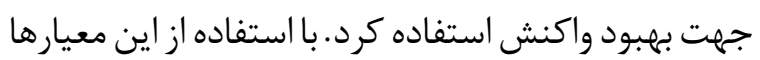

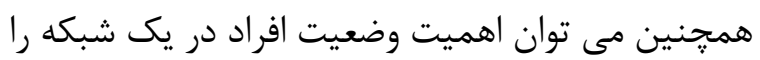

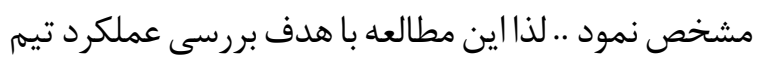

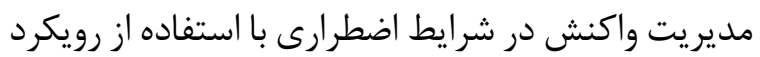

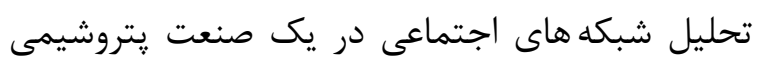

$$
\text { صورت گرفت. }
$$

Iran Occupational Health. 2021 (01 Apr);18: 7 
هيدروزن از محل اتصالات سيلندر در قسمت سيلندر ير

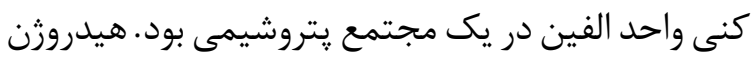

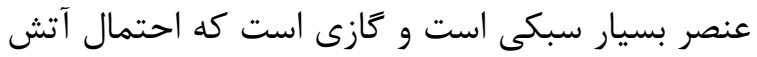

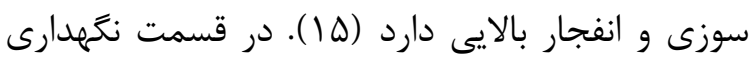

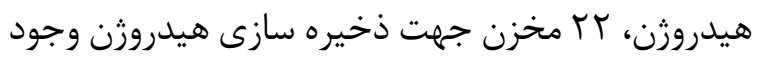

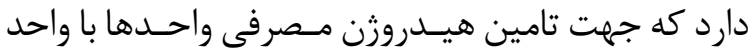

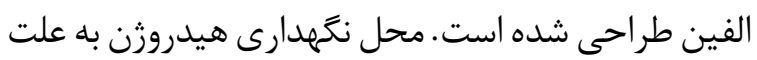

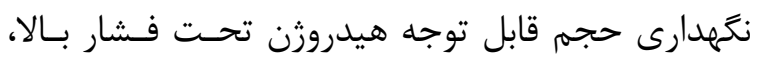

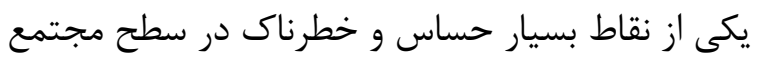

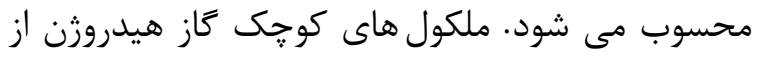

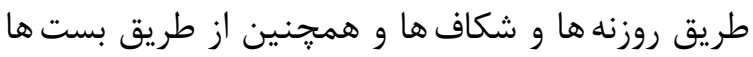

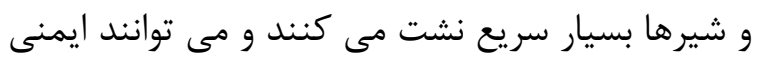

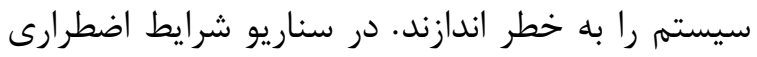

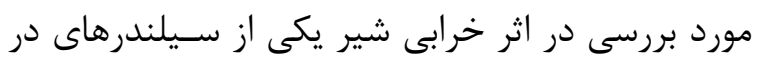

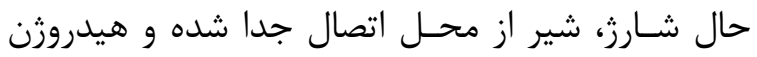

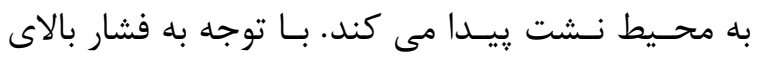

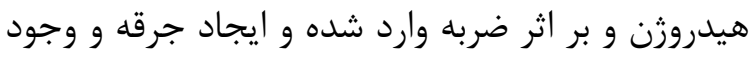

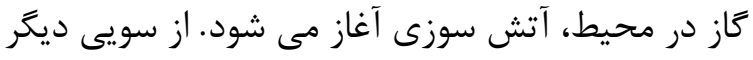

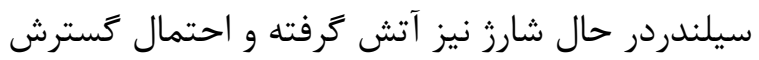

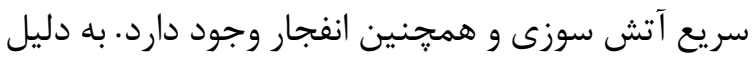

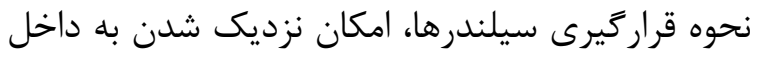

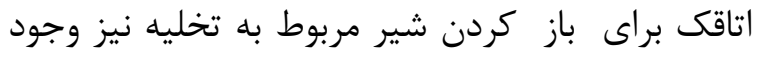

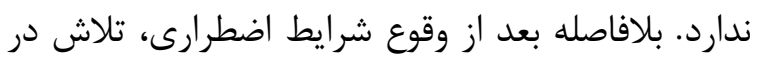

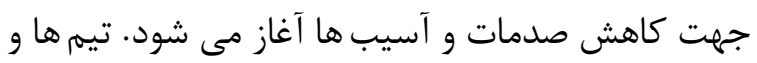

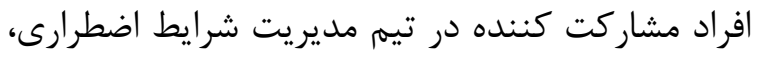

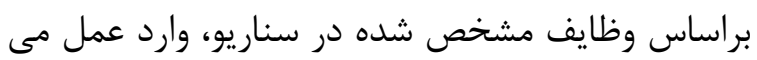

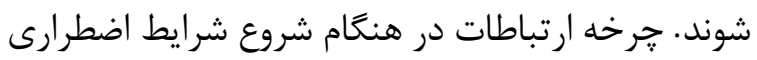

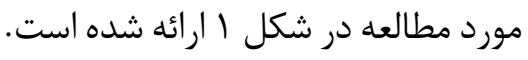

بافتنه ها

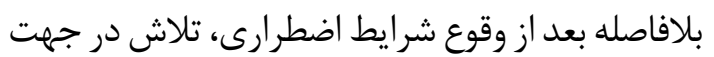

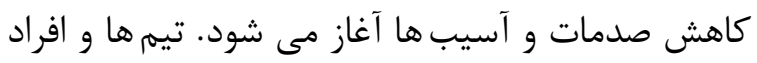

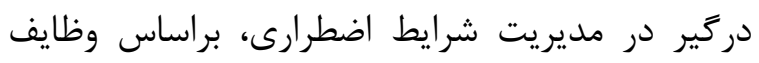

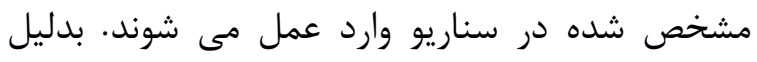

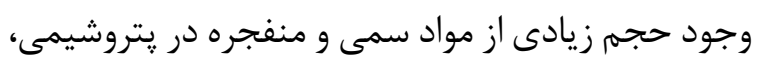

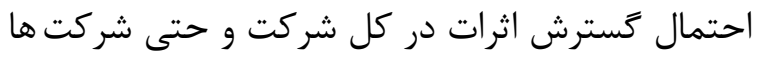

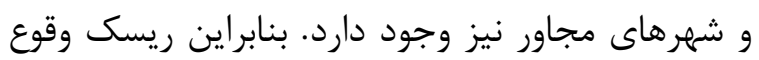

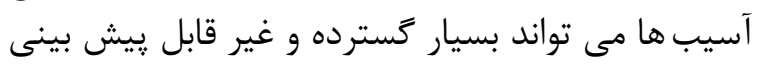

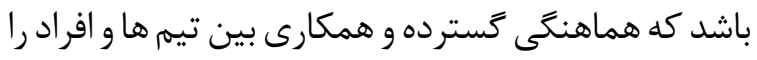

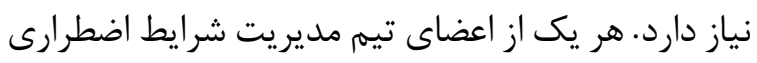

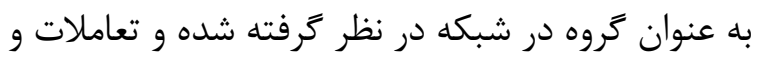
روابط آنها با يكديكر در طى شرايط اضطرارى در به عنه عنوان
قرار مى كيرد (r山) (r).

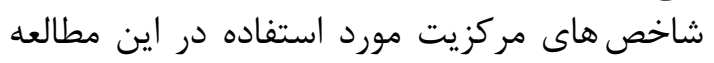

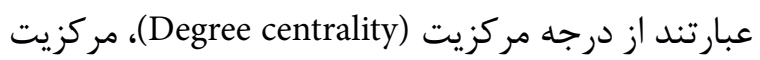

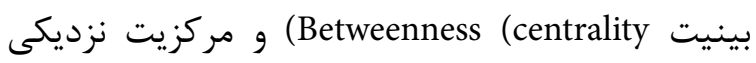
(Closeness Centrality)

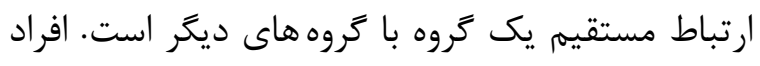

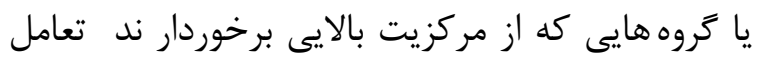

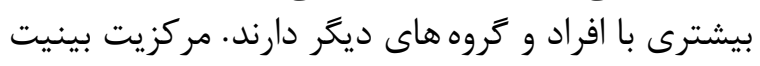

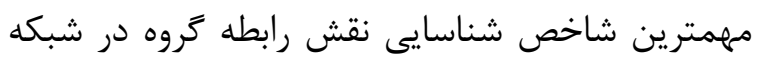

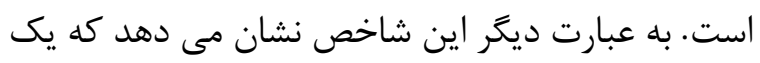

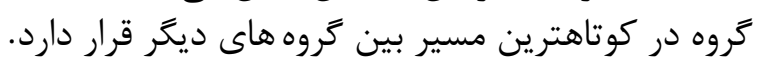

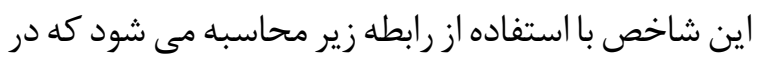
آن آن و كوتاهتر

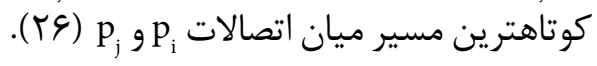

$C_{B}\left(p_{k}\right)=\sum_{i<1}^{n} \frac{g_{i j}\left(p_{k}\right)}{g_{i j}}$

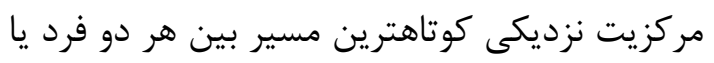

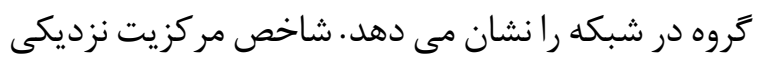

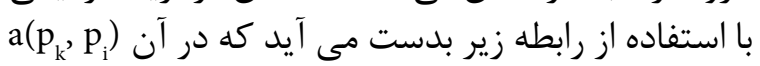
كوتاهترين مسير اتصال دو كره

$C_{c}\left(p_{k}\right)=\sum_{i=1}^{n} a\left(p_{i}, p_{k}\right)^{-1}$

تراكم يا جعالى نيز بيانكر اتصالات كل در شبكه است

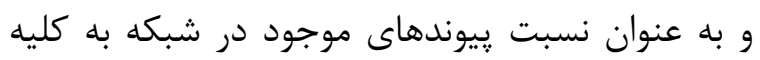

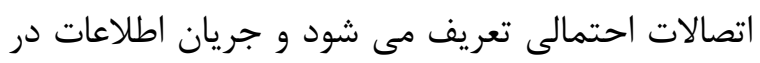

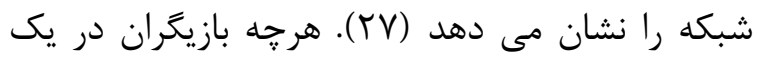

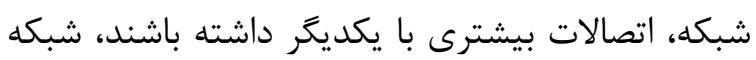

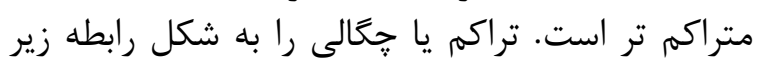

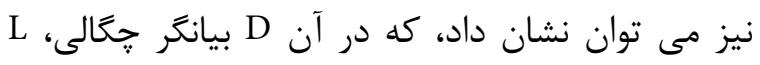

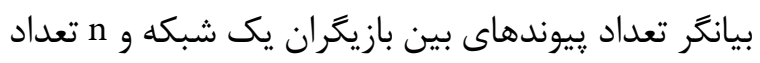

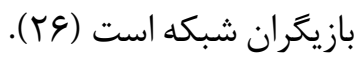
$D=\frac{L}{\frac{n(n-1)}{2}}$

تيم مديريت شر ايط اضطرارى شركت يتروشيمى مورد

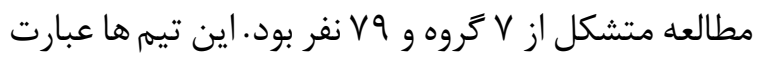

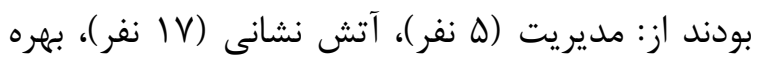

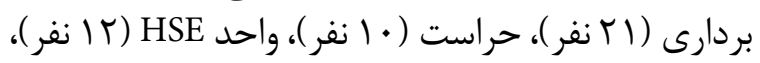

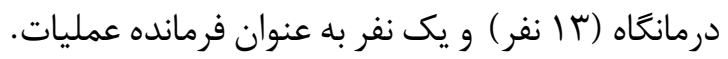
شرايط اضطرارى انتخاب شده در اين مطالعن نشان نشاند 


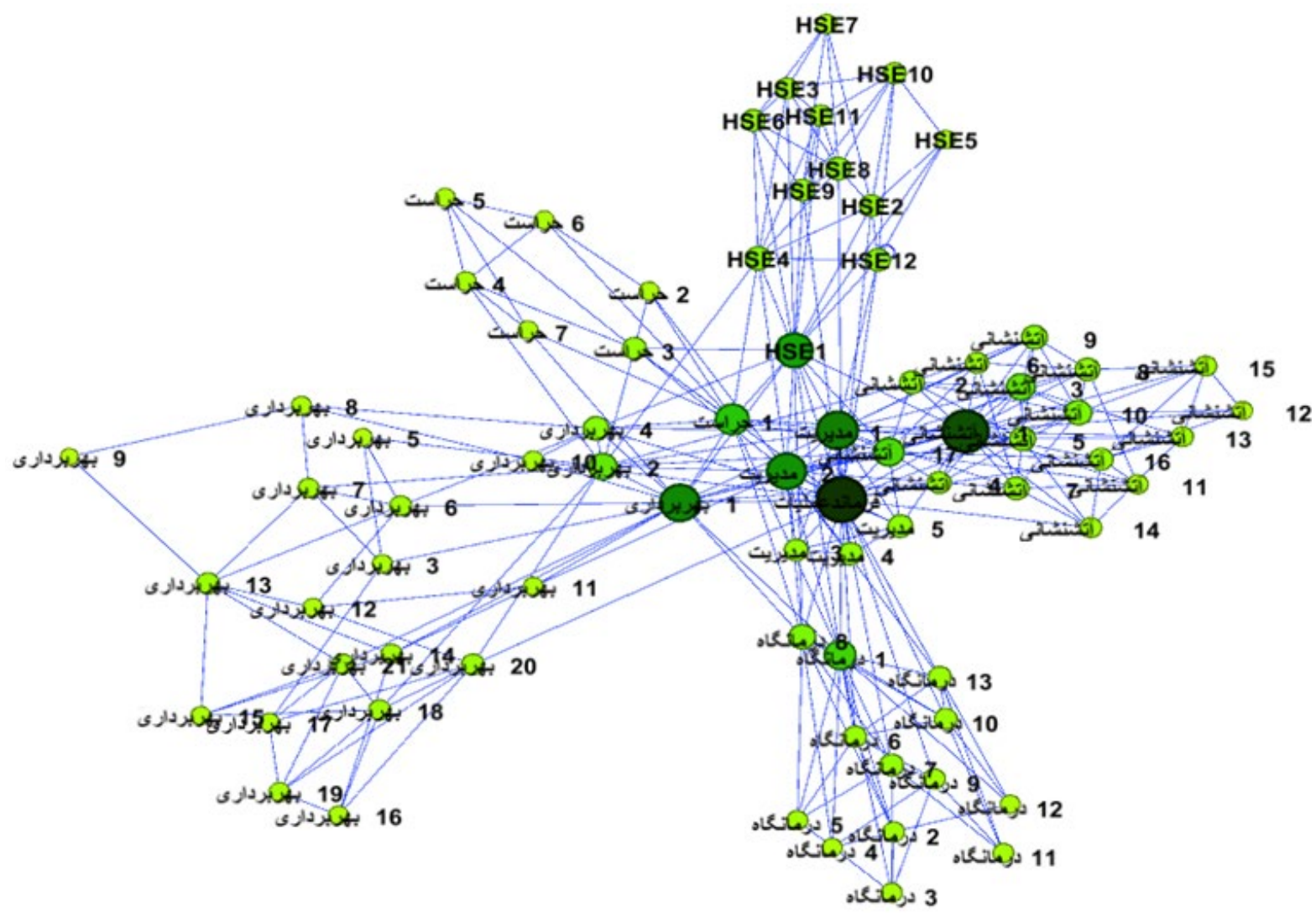

شكل ا. شبكه واكنش در شرايط اضطرارى (نرم افزار

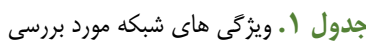

\begin{tabular}{|c|c|c|c|c|c|c|}
\hline ميانكين مركزيت نزديكى & ميانكين مركزيت بينيت & ميانكَين درجه مركزيت & ميانكَين تحَالى & تعداد يال & تعداد گره & 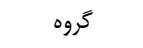 \\
\hline. $.9 \mathrm{~V}$ & Iro.V & $19 . t^{f}$ & 1 & 11 & $\Delta$ & مديريت \\
\hline$\cdot 9$ & VqI & To & 1 & & 1 & فرمانده عمليات \\
\hline$\cdot.)^{f}$ & $r \cdot . \cdot 1$ & 9.11 & س שq.. & sV & IV & آتش نشانى \\
\hline 苗 & 99.01 & 9.ru & אזוא. & DI & r) & بهره بردارى \\
\hline (\%) & rq. & ᄉ.VD & . ERI & il & it & HSE \\
\hline -.$r v$ & TEG & f.V & - .199 & r & 1. & حراست \\
\hline 的 & rT. $\cdot V$ & V.1 &..$^{.} \mathrm{V}$ & rV & r & درمانكاه \\
\hline
\end{tabular}

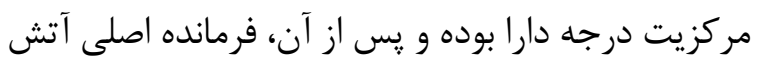

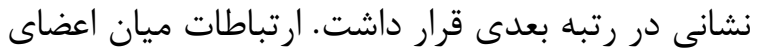

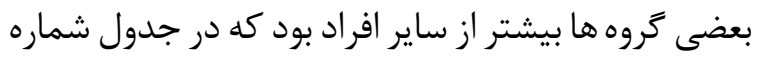
ا نيز اين تفاوت ها قابل ملاحظه است.

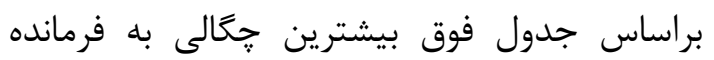

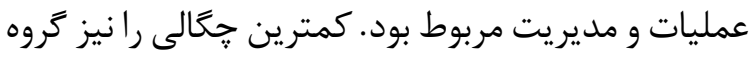

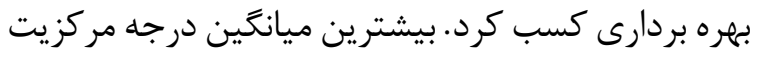

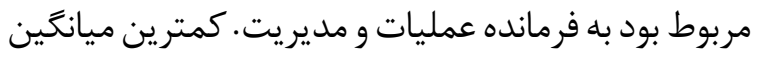
درجه مركزيت را كروه حراست كسب كرديت برديت بيشترين

$$
\text { يال ها مورد بررسى قراركرفت. }
$$

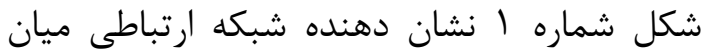

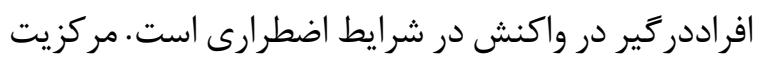

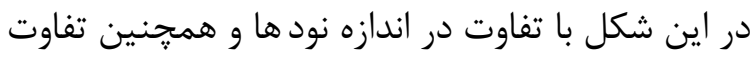

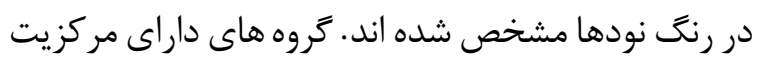

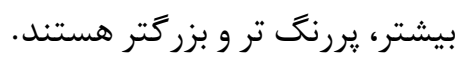

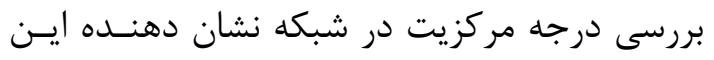

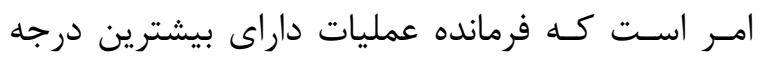

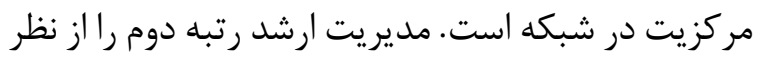


دهنـده اهميت گروه در نزديك نمودن مسـير ديخـر

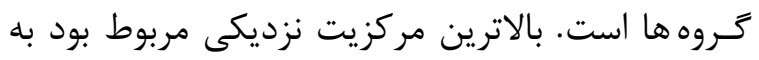

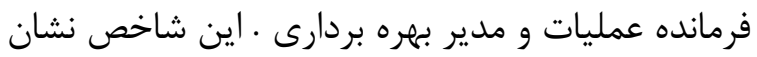

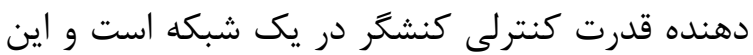

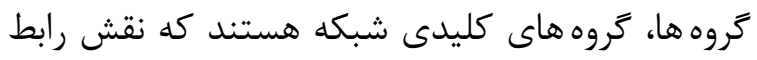

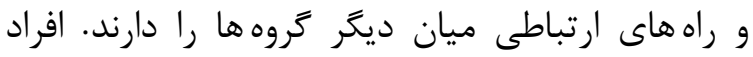

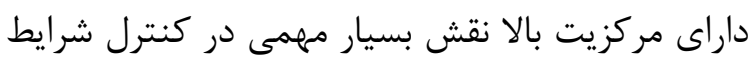

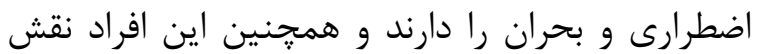

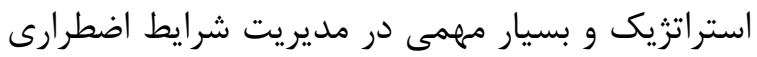

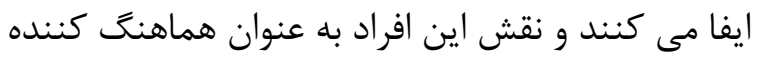

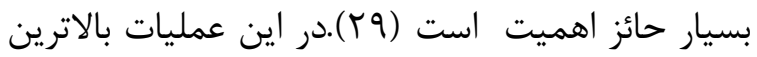

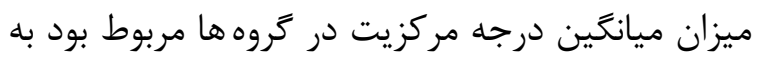

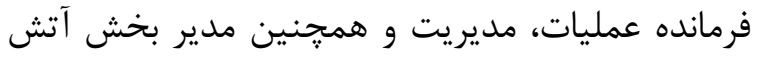

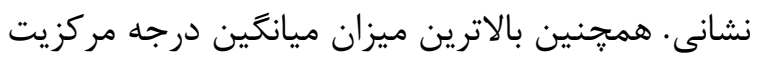

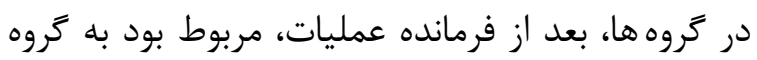

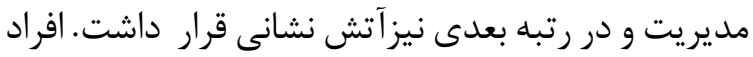

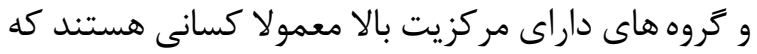

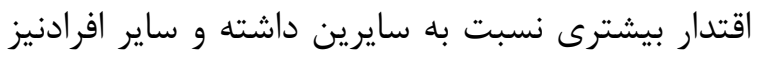

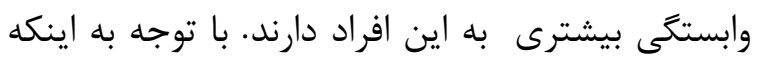

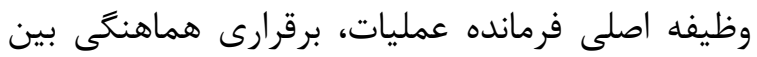

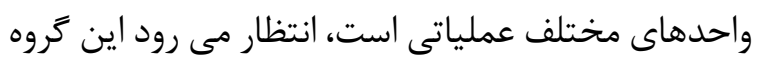

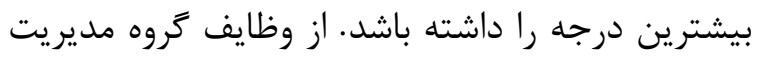

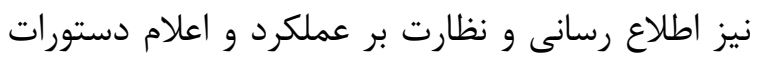

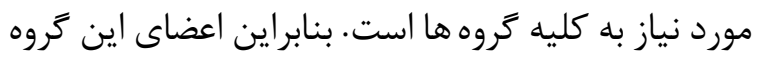

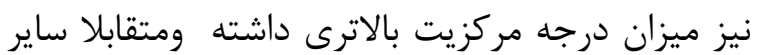

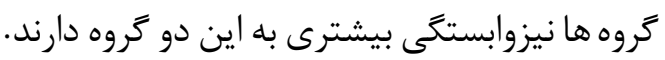

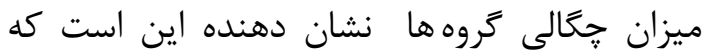

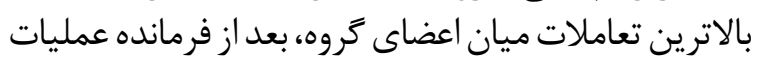

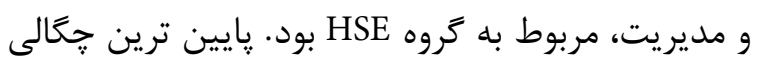

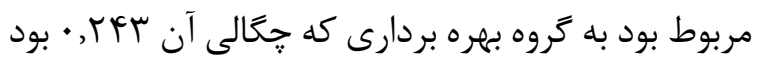

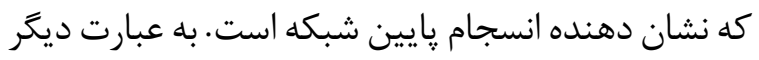

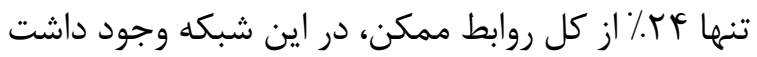

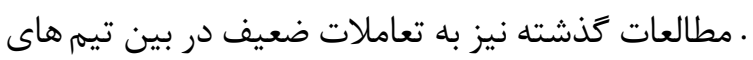

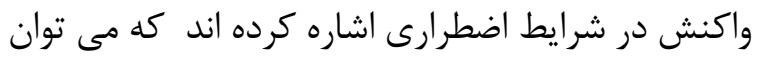

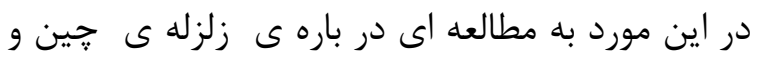

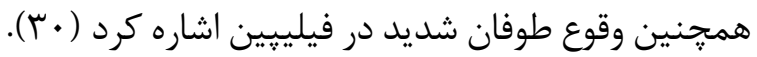

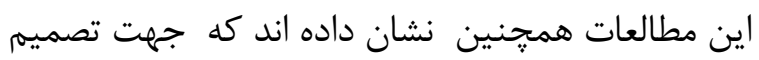

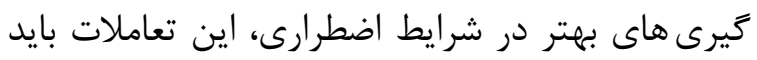

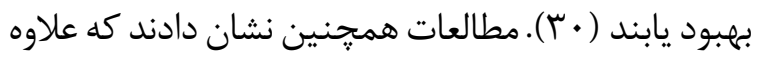

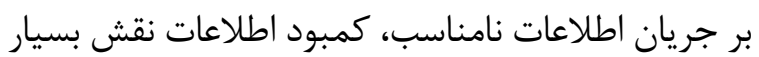

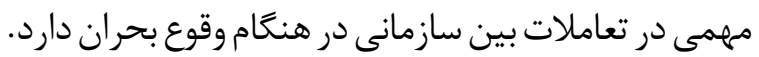

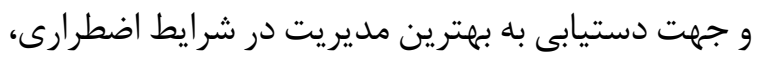

ميانكين مركزيت بينيت و همجنين ميانكين مركزيت

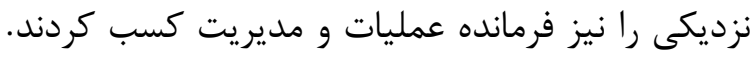

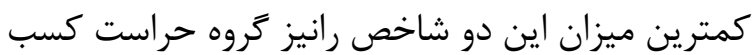

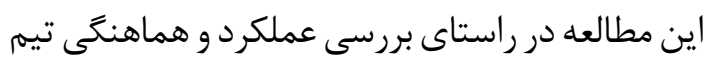

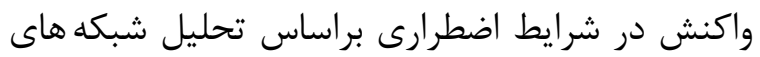

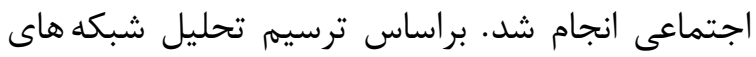

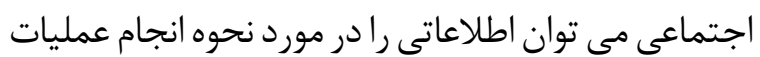

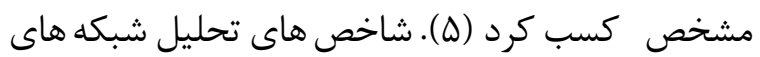

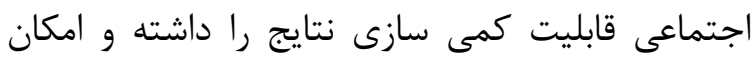

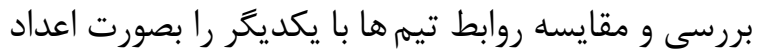

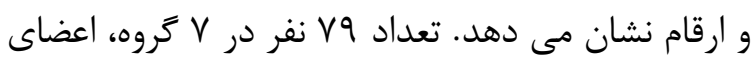

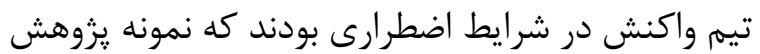

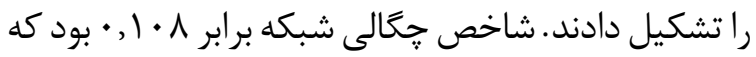

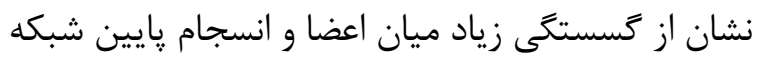

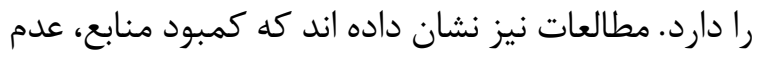

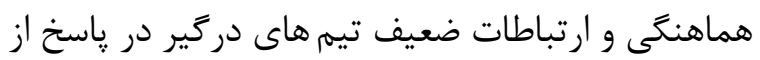

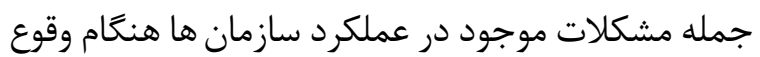

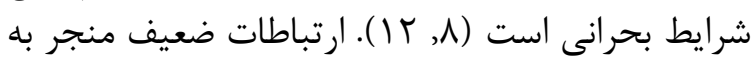

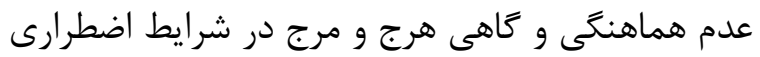

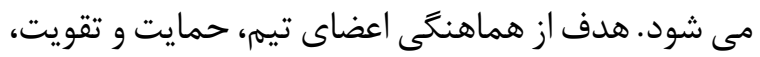

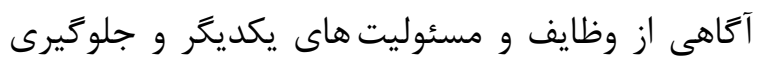

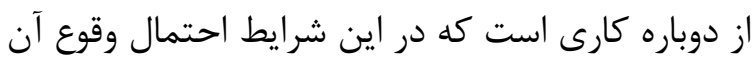

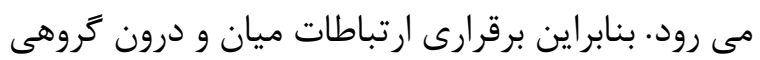

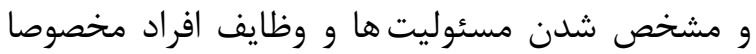

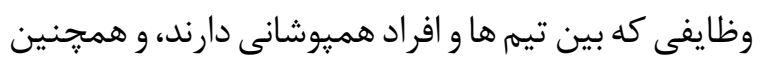

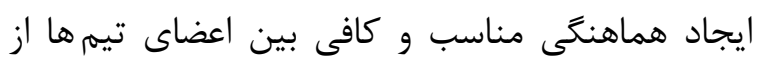

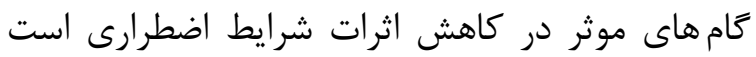

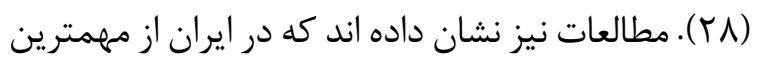

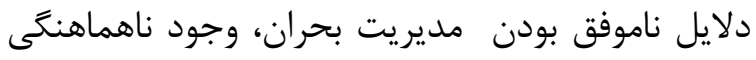

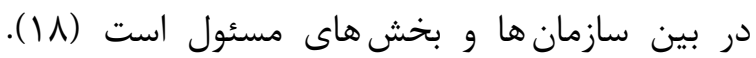

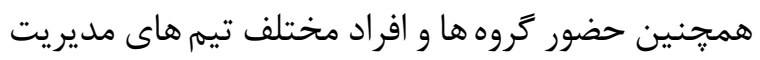

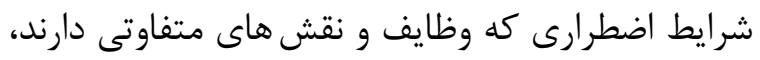

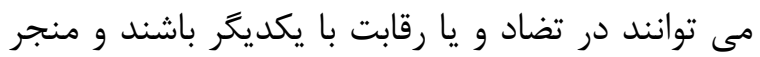

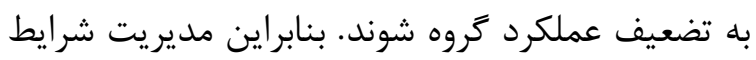

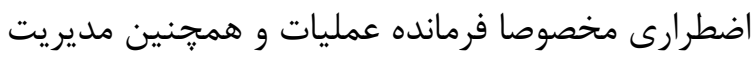

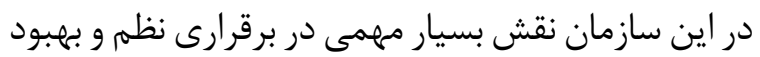

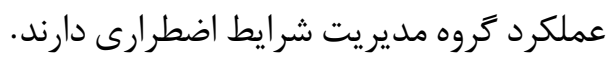

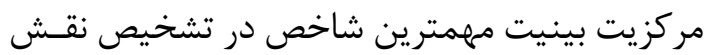

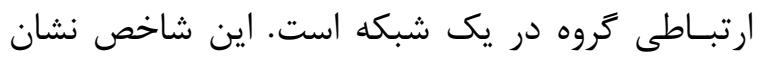


توسط نيروهاى امنيتى مى شوند، عدم اطلاع رسانى

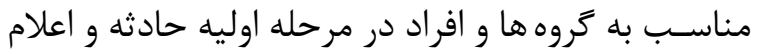

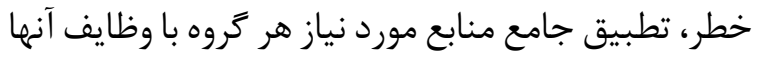

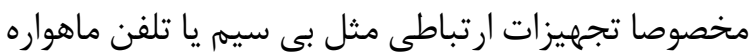

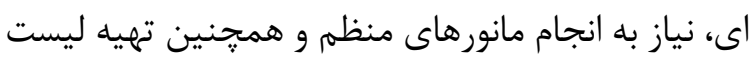

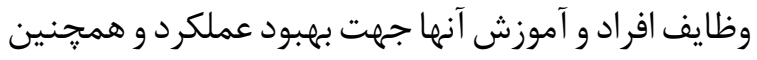

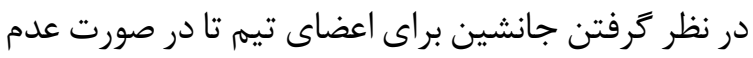
حضور يك فرد.

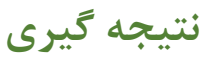

هماهنكى ميان افراد و تيمهاى دركير در شرايط

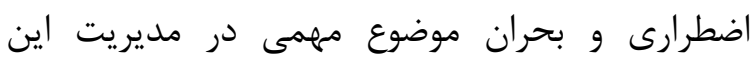

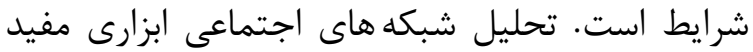

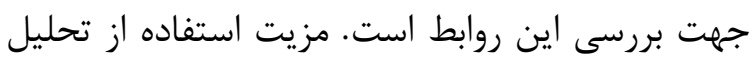

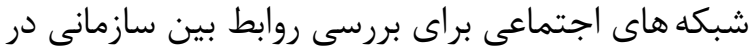

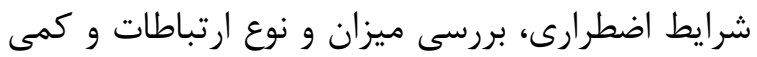

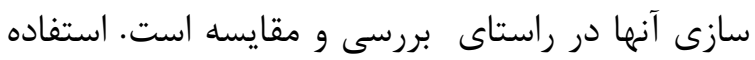

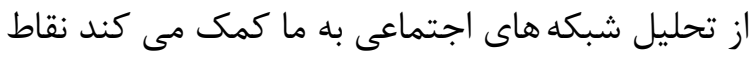

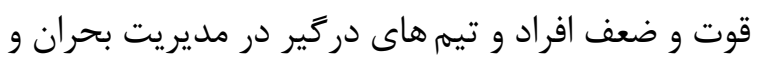

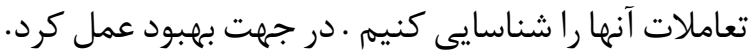

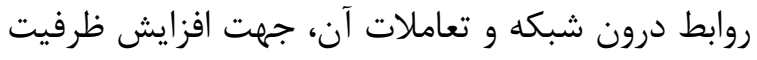

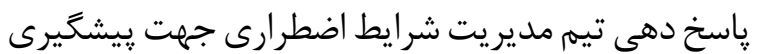

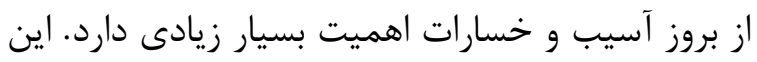

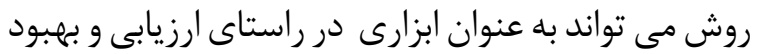

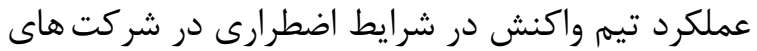
يتروشيمى مورد استفاده قرار كيرد.

$$
\text { تقدير و تشكر }
$$

اين مقاله بركرفته از يك طرح تحقيقاتى مصوب

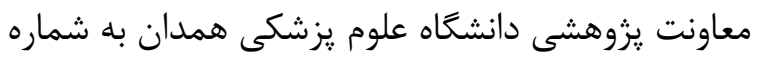

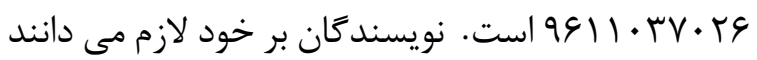

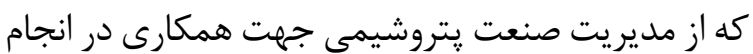
اين مطالعه قدردانى و تشكر نمايند.

\section{References}

1. Siqueira CE. Dependent convergence: the struggle to control petrochemical hazards in Brazil and the United States: Routledge; 2019.

2. Azadeh A, Fam IM, Garakani MM. A total ergonomic design approach to enhance the productivity in a complicated control system. Information Technology Journal. 2007;6(7):1036-42.

3. Mohammadfam I, Kamalinia M, Momeni M, Golmohammadi R, Hamidi Y, Soltanian A. Developing
نياز به اطلاعات دقيق، سريع و ارتباطات مناسب است

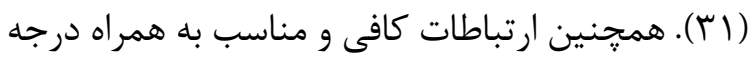

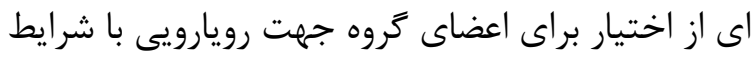

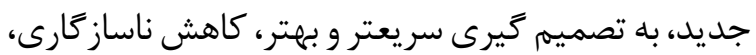

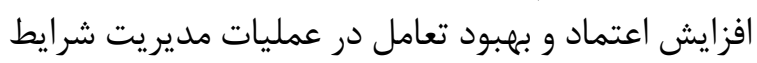

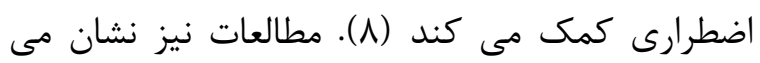

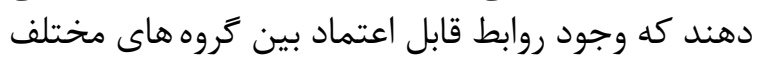

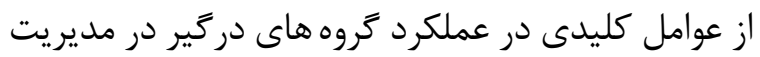

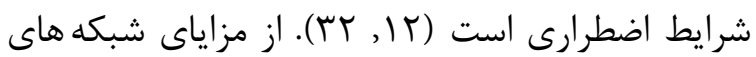

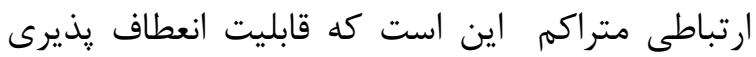

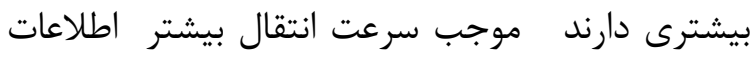

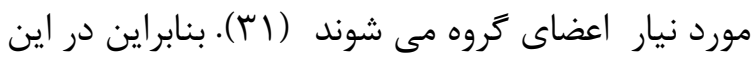

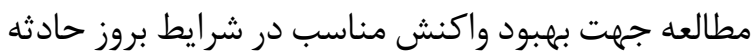

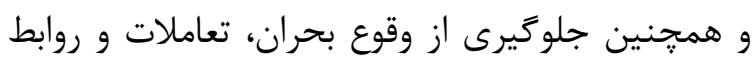

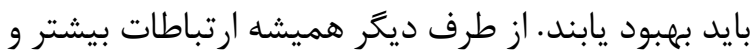

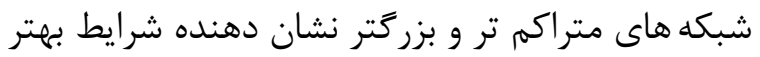

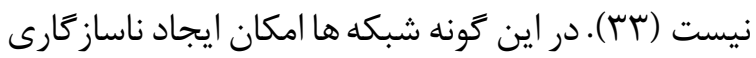

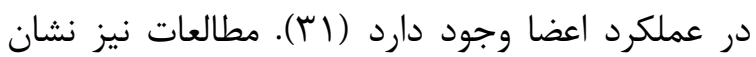

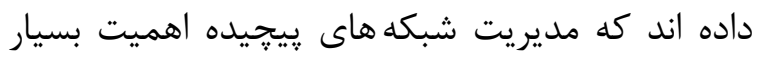

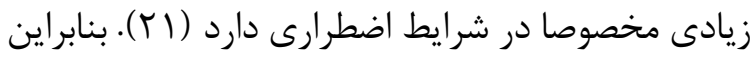

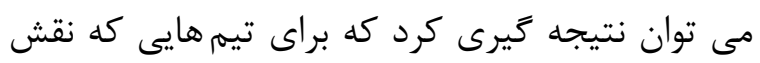

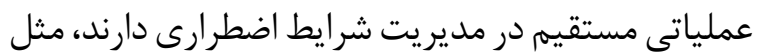

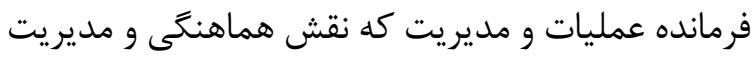

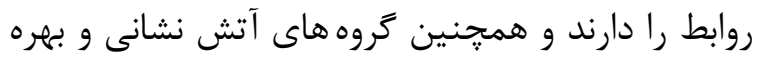

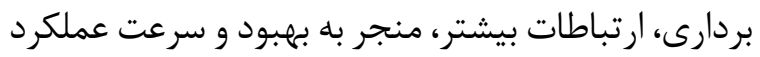

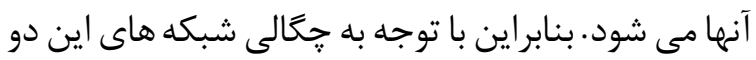

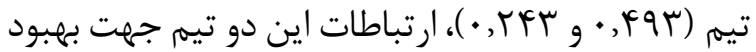

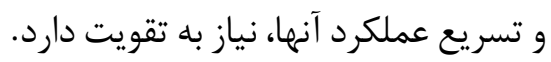

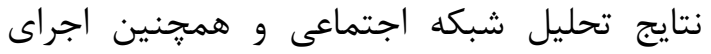

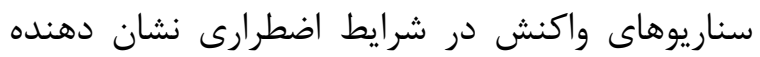

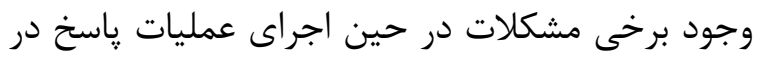

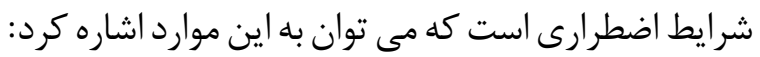

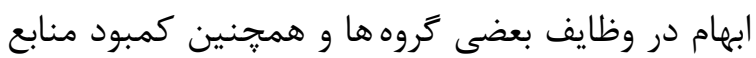

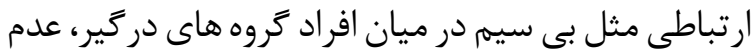

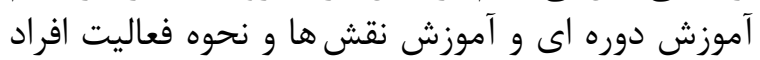

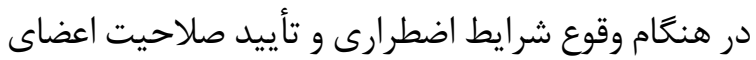

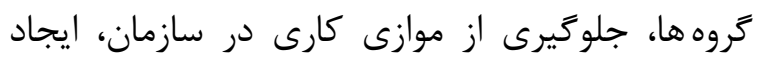

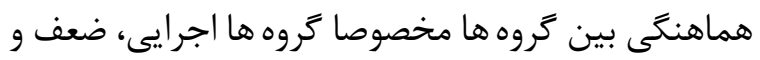

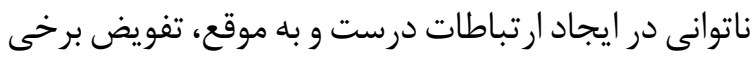

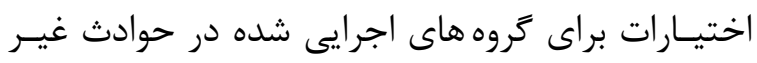

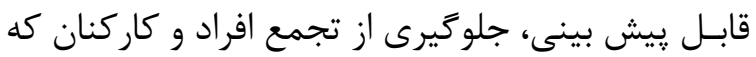

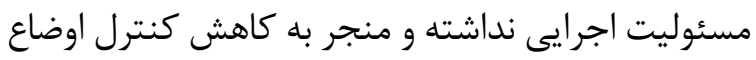


15. Mohammadian F. Survey and Comparison of sleep disorders in shift workers in the automotive industry. Iran Occupational Health. 2013;10(3):37-44.

16. Erickson PA. Emergency response planning: for corporate and municipal managers: Elsevier; 1999.

17. Johnson K. School crisis management: A hands-on guide to training crisis response teams: Hunter House; 2000.

18. Rabiee A, Ardalan A, Poorhoseini S. Assessment of coordination among lead agencies of natural disasters management in Iran. Hakim Research Journal. 2013;16(2):107-17.

19. Ray SJ. Strategic communication in crisis management: Lessons from the airline industry: Greenwood Publishing Group; 1999.

20. Zhang Y, Zou D, Zheng J, Fang X, Luo H. Formation mechanism of quick emergency response capability for urban rail transit: Inter-organizational collaboration perspective. Advances in Mechanical Engineering. 2016;8(6):1687814016647881.

21. Kapucu N, Garayev V. Designing, managing, and sustaining functionally collaborative emergency management networks. The American Review of Public Administration. 2013;43(3):312-30.

22. Houghton RJ, Baber C, McMaster R, Stanton NA, Salmon P, Stewart R, et al. Command and control in emergency services operations: a social network analysis. Ergonomics. 2006;49(12-13):1204-25.

23. Tang $\mathrm{P}$, Chen $\mathrm{H}$, Shao S. Examining the intergovernmental and interorganizational network of responding to major accidents for improving the emergency management system in China. Complexity. 2018;2018.

24. Choi SO, Brower RS. When practice matters more than government plans: A network analysis of local emergency management. Administration \& Society. 2006;37(6):651-78

25. Landherr A, Friedl B, Heidemann J. A critical review of centrality measures in social networks. Business \& Information Systems Engineering. 2010;2(6):371-85.

26. Abbasi A, Kapucu N. A longitudinal study of evolving networks in response to natural disaster. Computational and mathematical organization theory. 2016;22(1):47-70.

27. Ergün E, Usluel YK. An analysis of density and degreecentrality according to the social networking structure formed in an online learning environment. Journal of Educational Technology \& Society. 2016;19(4):34-46.

28. CAI N, WU J-b. Social Network Analysis on Denseness of Inter-organizational Networks in Industrial Clusters [J]. Journal of Zhejiang University (Humanities and Social Sciences). 2006;4.

29. Comfort LK, Ko K, Zagorecki A. Coordination in rapidly evolving disaster response systems: The role of information. American Behavioral Scientist. an integrated decision making approach to assess and promote the effectiveness of occupational health and safety management systems. Journal of Cleaner Production. 2016;127:119-33.

4. Abbasinia M, Kalatpour O, Motamedzadeh M, Soltanian A, Mohammadfam I. Dynamic Human Error Assessment in Emergency Using Fuzzy Bayesian CREAM. Journal of Research in Health Sciences. 2020;20(1).

5. Abbassinia $M$, Kalatpour $\mathrm{O}$, Motamedzade $\mathrm{M}$, Soltanian A, Mohammadfam I. Application of social network analysis to major petrochemical accident: interorganizational collaboration perspective. Disaster medicine and public health preparedness. 2020:1-8.

6. Azadeh A, Fam IM, Azadeh MA. Integrated HSEE management systems for industry: A case study in gas refinary. Journal of the Chinese Institute of Engineers. 2009;32(2):235-41.

7. Azadeh A, Mohammad Fam I. A framework for development of integrated intelligent human engineering environment. Information Technology Journal. 2006;5(2):290-9.

8. Mohammadfam I, Bastani S, Golmohamadi R, Saei A, Es-Haghi M. Applying social network analysis to evaluate preparedness through coordination and trust in emergency management. Environmental Hazards. 2015;14(4):329-40.

9. Mohammadfam I, Bastani S, Esaghi M, Golmohamadi R, Saee A. Evaluation of coordination of emergency response team through the social network analysis. Case study: oil and gas refinery. Safety and health at work. 2015;6(1):30-4.

10. Stojmenovic M, Lindgaard G. Social network analysis and communication in emergency response simulations. Journal of organizational computing and electronic commerce. 2014;24(2-3):236-56.

11. Abbasi A, Kapucu N. Structural dynamics of organizations during the evolution of interorganizational networks in disaster response. Journal of Homeland Security and Emergency Management. 2012;9(1).

12. Mohammadfam I, Bastani S, Es-haghi M, Golmohamadi $\mathrm{R}$, Saei A. Assessment of working interactions of emergency team members using social network analysis. Health and Safety at Work. 2014;4(3):37-48.

13. Harris JK, Clements B. Using social network analysis to understand Missouri's system of public health emergency planners. Public health reports. 2007;122(4):488-98.

14. Ghasemkhani M, Abbasinia M, Mahmoodkhani S, Aghaee H, Asghari M. Assessment of fatigue and its relationship with insomnia severity index in shift workers, fixed and rotating, Tehran rolling mills and steel production company. Iran Occupational Health. 2013;10(2):79-86. 
ارزيابى عملكرد تيم هاى واكنش در شرايط اضطرارى ...

Ebrahimipour V, Moghimbeigi A. Assessing resilience engineering based on safety culture and managerial factors. Process Safety Progress. 2012;31(1):17-8.

33. De Vries M, Kenis P, Kraaij-Dirkzwager M, Ruitenberg EJ, Raab J, Timen A. Collaborative emergency preparedness and response to cross-institutional outbreaks of multidrug-resistant organisms: a scenariobased approach in two regions of the Netherlands. BMC public health. 2019;19(1):52.
2004;48(3):295-313.

30. Huang Y. Modeling and simulation method of the emergency response systems based on OODA. Knowledge-Based Systems. 2015;89:527-40.

31. lateef Saeed NA, Zakaria NH, Sutoyo E. Team Performance in Flood Emergency Response: A Conceptual Model and Scale Development. International Journal of Integrated Engineering. 2018;10(6).

32. Shirali G, Mohammadfam I, Motamedzade M, 